\title{
Quantification and optimisation of lung ventilation SPECT images
}

\author{
Pernilla Norberg
}

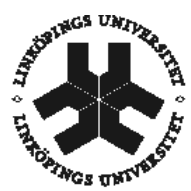

Linköping University

FACULTY OF HEALTH SCIENCES

Radiation Physics, Department of Medical and Health Sciences

Center for Medical Image Science and Visualization

Linköping University, Sweden

Linköping 2014 
(C) Pernilla Norberg, 2014

Cover: Pixlr.com edited human lung image. Original CT image from Petter Quick at CMIV.

\section{(c) (7) (9)}

This work (except Papers I and II) is licensed under the Creative Commons Attribution-NonCommercial 2.5 Sweden License. To view a copy of this license, visit http://creativecommons.org/licenses/bync/2.5/se/ or send a letter to Creative Commons, 444 Castro Street, Suite 900, Mountain View, California, 94041, USA.

Paper I has been reprinted with the permission of the copyright holder.

Printed in Sweden by LiU-Tryck, Linköping, Sweden, 2014

ISBN 978-91-7519-359-5

ISSN 0345-0082 
Be kind to yourself -Yogi tea 



\section{CONTENTS}

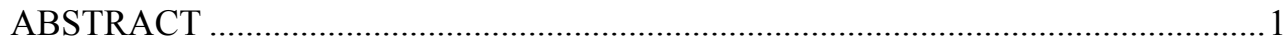

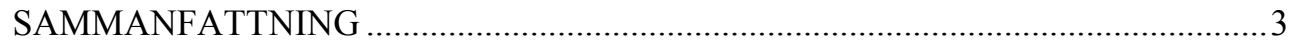

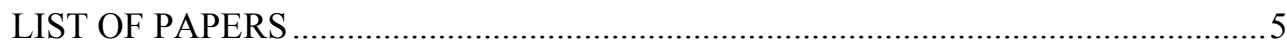

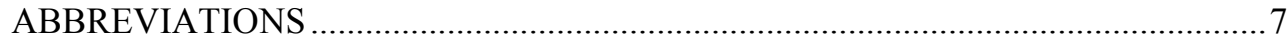

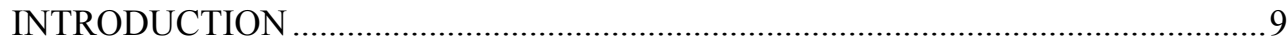

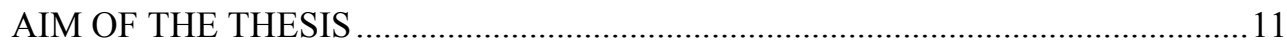

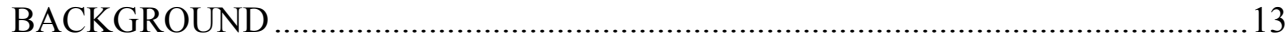

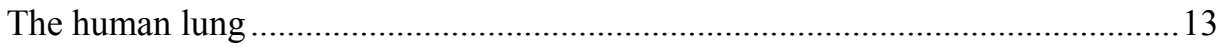

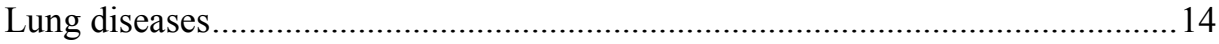

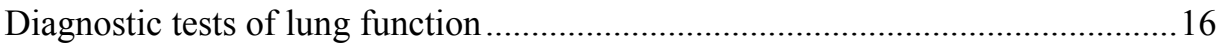

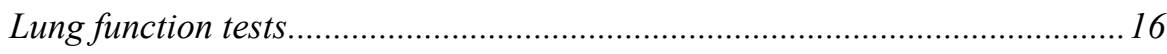

High resolution computed tomography, HRCT ......................................... 17

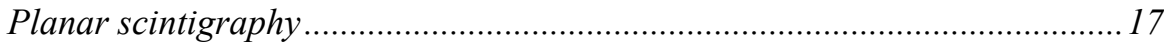

Single photon emission computed tomography, SPECT .............................. 17

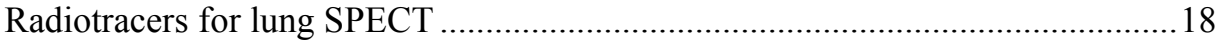

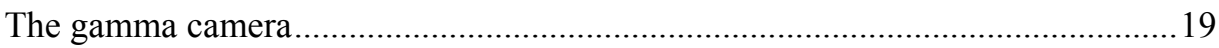

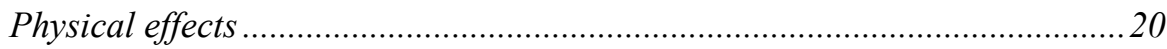

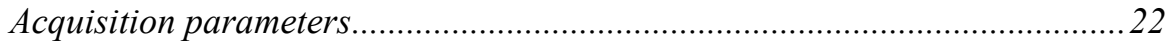

Monte Carlo simulations and phantoms .........................................................22

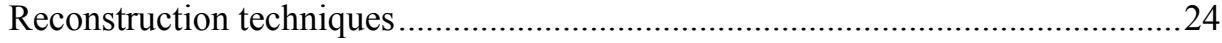

Filtered back projection, FBP .......................................................... 24

Ordered subset expectation maximisation, OSEM........................................ 24

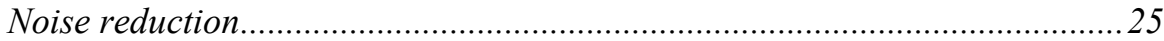

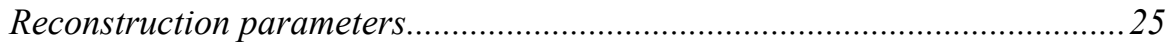

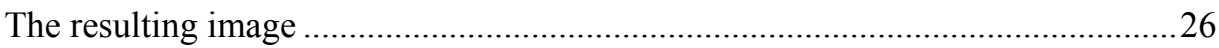

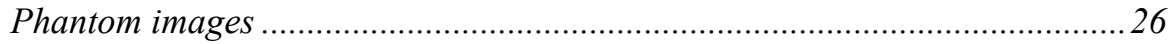

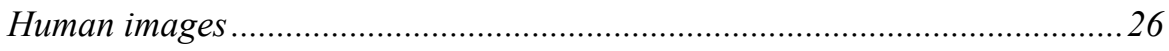

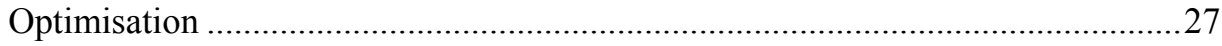

IMAGE AND COMPUTER PROCESSING ......................................................29 
EVALUATION OF RECONSTRUCTION ALGORITHM ....................................... 31

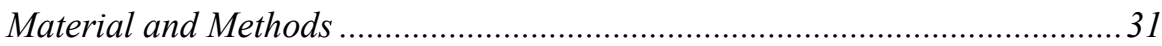

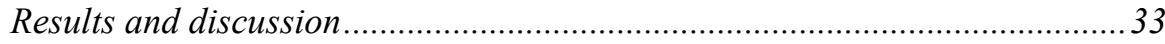

DEVELOPMENT AND OPTIMISATION OF THE CV $\mathrm{T}_{\mathrm{T}}-\mathrm{METHOD} \mathrm{...........................35}$

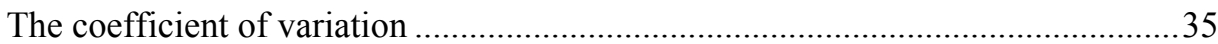

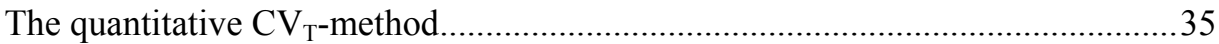

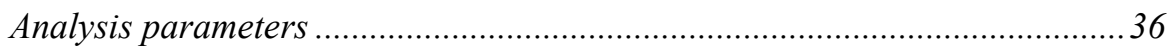

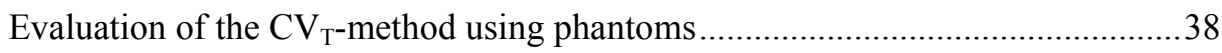

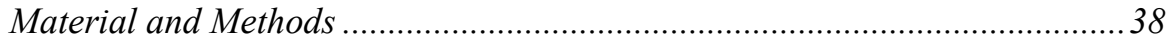

Results and discussion.......................................................................... 40

Optimisation of the included parameters when using the $\mathrm{CV}_{\mathrm{T}}-$ method.................42

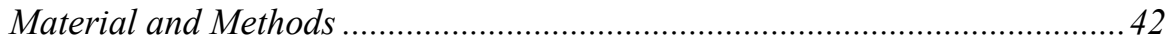

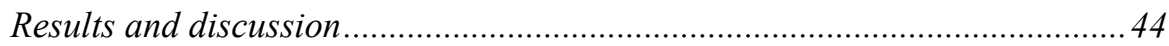

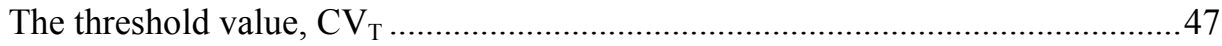

Volume of interest and kernel size ................................................................ 48

The compensation method.............................................................................49

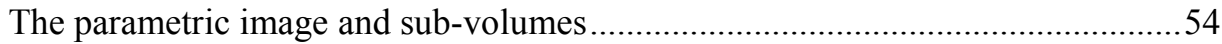

EVALUATION OF LUNG FUNCTION ON HUMAN SUBJECTS .........................57

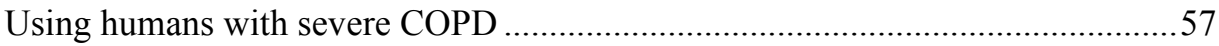

Material and Methods .............................................................................5 57

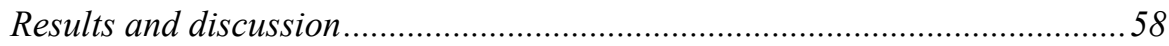

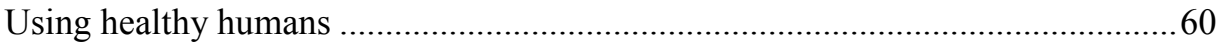

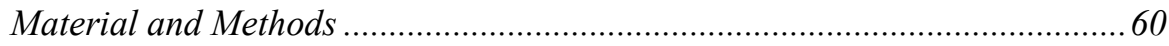

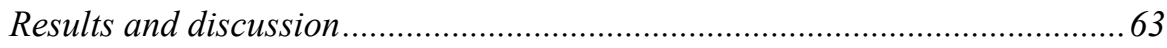

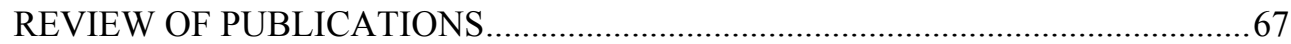

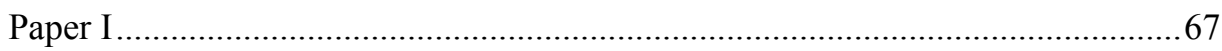

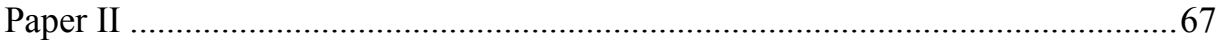

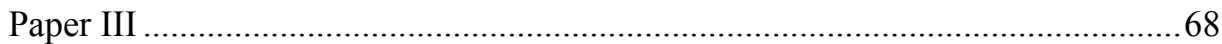

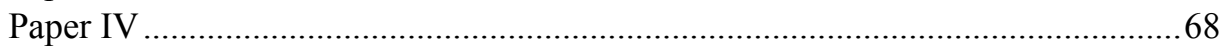

SUMMARY AND CONCLUSIONS .............................................................. 71

Future work and improvements ............................................................ 71

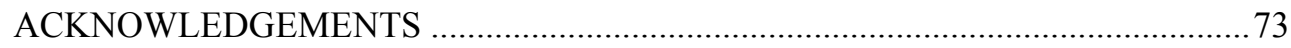

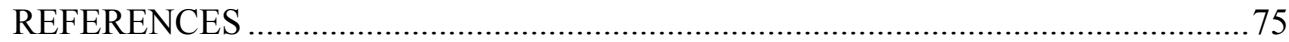




\section{ABSTRACT}

Currently, lung function tests are the gold standard for lung function measurements. Since the outcome of a lung function test is a summation of the status of the whole lung, significant changes in lung function may occur before a deviation from the norm can be identified. A method that can reliably detect lung abnormalities earlier in a disease process would therefore be beneficial. Regional differences in the lung are ideally studied by imaging methods. Heterogeneous ventilation in lungs of allergic individuals, cigarette smokers, asthmatics and chronic obstructive pulmonary disease (COPD) patients has been demonstrated using various imaging techniques such as single photon emission computer tomography, SPECT. The amount of heterogeneous ventilation is correlated to disease advancement. The $\mathrm{CV}_{\mathrm{T}}$-method, that measures heterogeneity using the coefficient of variation (CV) caused by lung function reduction in lung SPECT images, was developed and optimised. Lung function in patients and healthy volunteers was evaluated using the $\mathrm{CV}_{\mathrm{T}}$-method.

Monte Carlo simulated gamma camera projections were generated of activity distributions in two anthropomorphic phantoms. When comparing the two reconstruction algorithms, filtered back projection (FBP) and ordered subset expectation maximisation (OSEM), trade-off plots of spatial resolution, contrast and noise were used. Development and optimisation of the $\mathrm{CV}_{\mathrm{T}}$-method was performed using activity distributions mimicking various degrees of COPD. The $\mathrm{CV}_{\mathrm{T}}$-method itself was used when the optimal combination of acquisition, reconstruction and analysis parameter values was determined. The radioactive tracer ${ }^{99 \mathrm{~m}} \mathrm{Tc}-\mathrm{Tech}$ egas was used for the ventilation examination on human subjects.

OSEM resulted in higher spatial resolution in combination with lower noise level compared to FBP and was therefore chosen. The optimal parameter values found were a total number of counts in the projections of at least $3.6 \times 10^{6}$ and a low energy high-resolution collimator. The number of OSEM updates and cut-off frequency of the noise reduction filter depended on if the periphery of the lung was excluded or not. The $\mathrm{CV}_{\mathrm{T}}$-method showed to be capable of identifying early COPD in computer-simulated images $(\mathrm{p}<0.001)$. The $\mathrm{CV}_{\mathrm{T}}$-method was also capable of correctly identifying patients with severe COPD $(p<0.05)$. A compensation technique was implemented, making the heterogeneity values from healthy lung volumes of different subjects comparable. This adaptation made it possible to identify subjects who had normal lung function tests but with indications of conditions associated with ventilation disturbances. The results indicate that the present method has the capacity to identify minor lung function abnormalities earlier in a disease process than conventional lung function tests. 



\section{SAMMANFATTNING}

Lungfunktionstester (spirometri) är idag standardmetoden för att mäta lungors funktion. Dessa tester mäter dock hela lungans sammanvägda funktion och det kan därför krävas förhållandevis stora förändringar för att med säkerhet kunna identifiera någon lungfunktionsnedsättning. Det skulle vara fördelaktigt med en metod som identifierar nedsatt lungfunktion tidigare i sjukdomsutvecklingen för att kunna motivera åtgärder att bromsa sjukdomsförloppet. En bildgivande diagnostikmetod har fördelen att kunna lokalisera var i lungan funktionen är nedsatt. Med hjälp av olika bildgivande tekniker har heterogen eller ojämn lungventilation konstaterats hos allergiker, rökare, astmatiker och patienter med kronisk obstruktiv lungsjukdom (KOL). Graden av ojämn regional lungventilation samvarierar med graden av lungfunktionsnedsättning det vill säga graden av lungsjukdom. I detta arbete har $\mathrm{CV}_{\mathrm{T}}$-metoden utvecklats och optimerats. Metoden mäter ojämnhet i tredimensionella lungventilationsbilder med hjälp av variationskoefficienten $(\mathrm{CV})$. Bilden är resultatet från en SPECT-undersökning då ett radioaktivt spårämne har andats in. Lungfunktionen utvärderades hos både friska försökspersoner och patienter med hjälp av $\mathrm{CV}_{\mathrm{T}}$-metoden.

Radioaktivitetsfördelningar i två människoliknande fantom användes och den så kallade Monte Carlo-metoden utnyttjades för att i datorn simulera SPECT undersökningar. Två bildrekonstruktionsmetoder jämfördes; filtrerad bakåtprojektion (FBP) och iterativ rekonstruktion (ordered subset expectation maximisation, OSEM) och avvägningar mellan olika mått på bildkvalitet gjordes. Metodutveckling och optimering av $\mathrm{CV}_{\mathrm{T}}$-metoden utfördes genom att $\mathrm{i}$ fantomens lungor efterlikna olika grader av lungfunktionsnedsättning. Vid optimeringen bestämdes optimal kombination av ingående parametrars värden för att säkerställa att den i patienten upptagna radioaktiviteten tillsammans med SPECT-undersökningen och $\mathrm{CV}_{\mathrm{T}}$-metoden gav bästa möjliga resultat. Vid lung-SPECT-undersökningar av försökspersoner användes en radioaktiv kolgas (Technegas) som radioaktivt spårämne.

Bildrekonstruktionsmetoden OSEM gav högre skärpa och lägre brus jämfört med FBP och valdes därför. En relativt hög aktivitetsnivå och undersökning med hög upplösning gav optimalt resultat. $\mathrm{CV}_{\mathrm{T}}$-metoden visade sig kunna skilja på lindrig lungfunktionsnedsättning i människolikt lungfantom $(\mathrm{p}<0.001)$. Metoden kunde också identifiera patienter med allvarlig KOL $(p<0.05)$. En kompensationsmetod utvecklades för att bättre kunna jämföra resultat mellan friska försökspersoner. Därefter kunde personer identifieras med normala lungfunktionstester men med indikation på lungventilationsstörning. Resultaten tyder på att $\mathrm{CV}_{\mathrm{T}}$-metoden kan identifiera smärre lungfunktionsnedsättning tidigare i sjukdomsförloppet än traditionellt lungfunktionstest. 



\section{LIST OF PAPERS}

The present thesis is based on the following papers. They are referred to in the text by their capital Roman numerals.

I. Evaluation of reconstruction techniques for lung single photon emission tomography: A Monte Carlo study. Pernilla Norberg, Björn Bake, Lars Jacobsson, Gudrun Alm Carlsson and Agnetha Gustafsson. Nuclear Medicine Communications 2007,28:929-936.

II. Quantitative lung-SPECT applied on simulated early COPD and humans with advanced COPD. Pernilla Norberg, Lennart H Persson, Gudrun Alm Carlsson, Björn Bake, Magnus Kentson, Michael Sandborg and Agnetha Gustafsson. EJNMMI Research 2013 3:28, doi:10.1186/2191-219X-3-28 $818^{1}$.

III. Optimisation of quantitative lung SPECT applied to early COPD: a Monte Carlo-based analysis. Pernilla Norberg, Anna Olsson, Gudrun Alm Carlsson, Michael Sandborg and Agnetha Gustafsson. Submitted to Physics in Medicine and Biology December 2013.

IV. Does quantitative lung SPECT detect lung abnormalities earlier than lung function tests? Results of a pilot study. Pernilla Norberg, Lennart H Persson, Gudrun Alm Carlsson, Birgitta Schmekel, Karl Wahlin, Michael Sandborg and Agnetha Gustafsson. Manuscript submitted to EJNMMI Research April 2014.

\section{Contributions:}

I. The project was initiated by B. Bake and L. Jacobsson. I planned the work together with A. Gustafsson and G. Alm Carlsson. I performed the simulations, reconstructions and analysis. I wrote the article with assistance from B. Bake, L. Jacobsson, G. Alm Carlsson and A. Gustafsson. I was the corresponding author.

II. The project was initiated by A. Gustafsson and me. I planned the work together with A. Gustafsson and L. Persson. Healthy subjects were recruited by me and patients by L. Persson. I designed the quantitative image analysis method and performed the simulations, reconstructions, image- and statistical analysis. I wrote the article with assistance from L. Persson, G.

\footnotetext{
${ }^{1}$ Open Access, Creative Commons Attribution License (http://creativecommons.org/licenses/by/2.0)
} 
Alm Carlsson, B. Bake, M. Kentson, M. Sandborg and A. Gustafsson. I was the corresponding author.

III. The project was initiated by me and A. Gustafsson. I planned the work together with A. Gustafsson, M. Sandborg and A. Olsson. I performed the simulations, reconstructions, image- and statistical analysis. I wrote the article with assistance from G. Alm Carlsson, A. Olsson, M. Sandborg and A. Gustafsson. I am the corresponding author.

IV. The project was initiated by me, A. Gustafsson, L. Persson and B. Schmekel. I planned the work together with them. Healthy subjects were recruited by me and patients by L. Persson. Lung function tests were interpreted by B. Schmekel. K. Wahlin made the statistical calculations, reviewed the model adjustments and interpreted the results. I performed the reconstructions and image analysis. I wrote the article with assistance from L. Persson, G. Alm Carlsson, B. Schmekel, Karl Wahlin, M. Sandborg and A. Gustafsson. I am the corresponding author.

Peer reviewed conference abstracts. They are referred to in the text by their lower case Roman numerals:

i. P. Norberg, B. Bake, M. Sandborg, G. Alm Carlsson, A. Gustafsson. "The potential of lung SPECT in identifying humans with early stages of COPD; a Monte Carlo-based analysis". Annual congress of the EANM, Birmingham, October, 2011

ii. P. Norberg, H.L. Persson, G. Alm Carlsson, B Bake, M. Kentson, M. Sandborg and A. Gustafsson. "The potential of quantitative lung SPECT in identifying humans with COPD using the CVT-method; a Pilot Study of advanced disease". Annual congress of the EANM, Milano, October, 2012.

iii. P. Norberg, M. Sandborg, G. Alm Carlsson, A. Gustafsson, H.L. Persson, B. Bake, M. Kentson. "Quantitative lung-SPECT applied on simulated early COPD and humans with advanced COPD”. 3rd annual conference on Medical Physics, Djurönäset, November, 2012.

Other related publications not included in the thesis:

P. Norberg, $\mathrm{CV}_{\mathrm{T}}$-metoden, -ny metod för identifiering av lindrig lungfunktionsnedsättning, BestPractise, mars (NR 5) 2014 (Årgång 2).

Keywords: SPECT, Quantitative evaluation, Lung, Lung diseases, Monte Carlo method, Image reconstruction, Computer Assisted Image Analysis 


\section{ABBREVIATIONS}

$\mathrm{AUC}\left(\mathrm{CV}_{\mathrm{T}}\right)$ The area under the curve for $\mathrm{CV}$ values greater than the threshold value

CDR Collimator detector response

CI Confidence interval

COPD Chronic obstructive pulmonary disease

CT Computed tomography

CV The coefficient of variance

$\mathrm{CV}_{\mathrm{T}} \quad$ The threshold value of $\mathrm{CV}$

DLCOc Diffusion capacity for carbon monoxide compensated for haemoglobin concentration in the blood

ESSE Effective source scatter estimation

FBP Filtered back-projection

$\mathrm{FEV}_{1} \quad$ Forced expiratory volume measured over one second

FVC Forced vital capacity

FWHM Full width at half maximum

HRCT High resolution computer tomography

LEGP Low energy general purpose

LEHR Low energy high resolution

MLEM Maximum likelihood expectation maximisation

MRI Magnetic resonance imaging

NMSE Normalised mean square error

OSEM Ordered subset expectation maximisation

PET Positron emission tomography

RV Residual volume

SPECT Single photon emission computed tomography

TLC Total lung capacity

TV Tidal volume

VC Vital capacity 



\section{INTRODUCTION}

Heterogeneous, uneven or patchy ventilation in lungs of allergic individuals, cigarette smokers, asthmatics and COPD patients have been demonstrated using various imaging techniques such as positron emission tomography (PET) (Tgavalekos et al., 2007; Venegas et al., 2005), magnetic resonance imaging (MRI) (Altes et al., 2001; Samee et al., 2003; Emami et al., 2013), planar scintigraphy images (Sovijarvi et al., 1982) and SPECT (Jogi et al., 2011). The amount of heterogeneous ventilation is correlated to disease advancement (Hedenstierna, 2000). These patients suffer from narrow and/or closed airways to varying degree. Narrowing of the airways is caused by inflammation, secretions and the shortening of muscle fibres around the bronchial walls, which obstructs airflow. Emami et al. (Emami et al., 2013) showed that asymptomatic smokers had a more heterogeneous ventilation distribution compared to healthy nonsmokers. Patchiness can be caused by narrowing of both larger and smaller airways. Sovijarvi et al. (Sovijarvi et al., 1982) suggested that although larger asthmatic airways are dilated by isoprenaline inhalations, residual bronchial obstruction may still remain in some smaller airways, maintaining a heterogeneous distribution. Furthermore, Tgavalekos et al. (Tgavalekos et al., 2007) concluded that the heterogeneous and patchy distribution of ventilation in asthma patients is a manifestation of the complex behaviour of the airway system, rather than the independent behaviour of individual airways. Closure of the airways by air trapping occurs when the small airways, the bronchioles, collapse. According to Özer et al. (Ozer et al., 2005), air trapping can be present even before lung function tests reveal abnormal results, or pulmonary symptoms become apparent. Possible causes of air trapping are aging, smoking and various obstructive diseases such as asthma (Ozer et al., 2005; Samee et al., 2003). The severity of air trapping has been shown to increase with age and smoking (Lee et al., 2000).

Currently lung function tests are the gold standard for lung function measurements. Since the outcome of a lung function test is a summation of the status of the whole lung, significant changes in lung function may occur before a deviation from the normal can be identified. A method that can reliably detect lung abnormalities earlier in the disease process would therefore be beneficial. One benefit is prevention of further lung degeneration earlier than previously possible. With a method able to identify small changes in lung function, various treatment strategies can be evaluated. Such method could also be a tool for identification of mild COPD when lung function tests and high resolution computed tomography (HRCT) couldn't give a clear answer.

$\mathrm{Xu}$ presented in 2001 (Xu et al., 2001a), regional heterogeneity measurements determined by calculating the coefficient of variation (CV) in small ele- 
ments of the lung. Heterogeneity maps and frequency curves were generated based on the CV values from ventilation SPECT images of nine emphysematous patients, nine healthy smokers and nine healthy non-smokers. The SPECT images were reconstructed by filtered back projection (FBP) with attenuation compensation. The $\mathrm{CV}$ values were calculated for $2 \times 2 \times 1 \mathrm{~cm}^{3}$ elements in four to five transversal SPECT slices (with $3.5 \mathrm{~cm}$ spacing between the slices) for each subject. Based on the pooled mean CV for each subject he found a significant difference between patients and smokers and between patients and non-smokers but not between smokers and non-smokers. Frequency curves of CV values for the non-smokers, a deviant smoker and a patient, illustrated the increased number of high $\mathrm{CV}$ value elements with increased disease advancement.

The outcome of the quantitative method by Xu could be improved by using a more modern reconstruction technique including compensating for attenuation as well as scatter and distance dependence. The quantitative method could be based on all voxels in the lung and not be limited to selected slices and elements. The acquisition, reconstruction and analysis parameter values used could be optimised to provide the best chance for the method to be successful in its task. Differentiation between healthy non-smokers and healthy smokers might then be possible, i.e. the method could become more sensitive to heterogeneities than lung function tests. 


\section{AIM OF THE THESIS}

The overall objective of this work was to create a method that can identify minor lung function abnormalities, earlier in a disease process than can be made by lung function tests. My work towards this objective can be divided into the following specific aims

- Evaluation of reconstruction methods for subjective visual evaluation of lung SPECT images.

- Improving the methodology introduced by Xu (Xu et al., 2001a) of extracting CV values out of a lung ventilation SPECT image.

- Evaluating the ability of the $\mathrm{CV}_{\mathrm{T}}$-method to differentiate between uniform and heterogeneous ventilation distributions using a anthropomorphic phantom.

- Optimising the acquisition, reconstruction and quantitative analysis with the $\mathrm{CV}_{\mathrm{T}}$-method in a anthropomorphic phantom simulating mild COPD.

- Improving the $\mathrm{CV}_{\mathrm{T}}$-method by creating and implementing a compensation procedure, to account for subject-to-subject variations of uptake.

- Evaluating the ability of the $\mathrm{CV}_{\mathrm{T}}$-method to differentiate between healthy volunteers and patients with severe COPD.

- Evaluating the potential of the improved $\mathrm{CV}_{\mathrm{T}}$-method to identify minor lung function abnormalities prior to that of conventional lung function tests. 



\section{BACKGROUND}

\section{The human lung}

The principal function of the respiratory system is to transport oxygen from the atmosphere into the blood and remove carbon dioxide (Figure 1). This exchange of gases takes place in the millions of tiny, exceptionally thin-walled air sacs called alveoli. This function can be disordered, mainly by diseases in the bronchial tubes and lungs.

The bronchial tree has on average 23 generations of branches between the mouth and the alveoli (West, 1991). Each branching results in narrower, shorter and more numerous tubes. The walls of the larger tubes, the bronchi, contain cartilage which gives them their cylindrical shape and supports them. The cartilage prevents the tubes from possible collapse. The first branches that no longer contain cartilage are termed bronchioles. The walls of the bronchioles contain smooth muscles which can increase or decrease the diameter of the bronchioles. Bronchi and bronchioles without alveoli make up the conductive airways and their function is to lead the inhaled air to the gas exchanging regions of the lung (West, 1991). Approximately the last ten generations (West, 1991; Bake, 2000) of the bronchial tree have direct contact with alveoli and this part of the lung is known as the respiratory zone. Down the respiratory zone the total cross sectional area of the airways increase enormously, because of the large number of branches, and the forward velocity of the inhaled air becomes very small (West, 1991). The average diameter of the smallest bronchiole, is $0.15-0.2 \mathrm{~mm}$ (Laga et al., 2008) and the diameter of an alveoli is about $0.30 \mathrm{~mm}$ (West, 1991). The total alveolar area is about $70-80 \mathrm{~m}^{2}$ (Bake, 2000). The lung tissue (parenchyma) consists of alveoli and the thin-masked net of tiny blood vessels surrounding them. The walls between the alveoli and the blood vessels are so thin that oxygen and carbon dioxide diffuse through the walls. This is where the red blood cells in the bloodstream receive oxygen from, and the blood gives away carbon dioxide to, the inhaled air.

Ventilation of the lung is defined as the exchange of air between the atmosphere and alveoli. Perfusion of the lung is the distribution of blood in the vessels. At rest, in a normal adult, approximately four litres of environmental air enter and leave the alveoli per minute, while five litre of blood flow through the pulmonary blood vessels (Vander et al., 1994). 


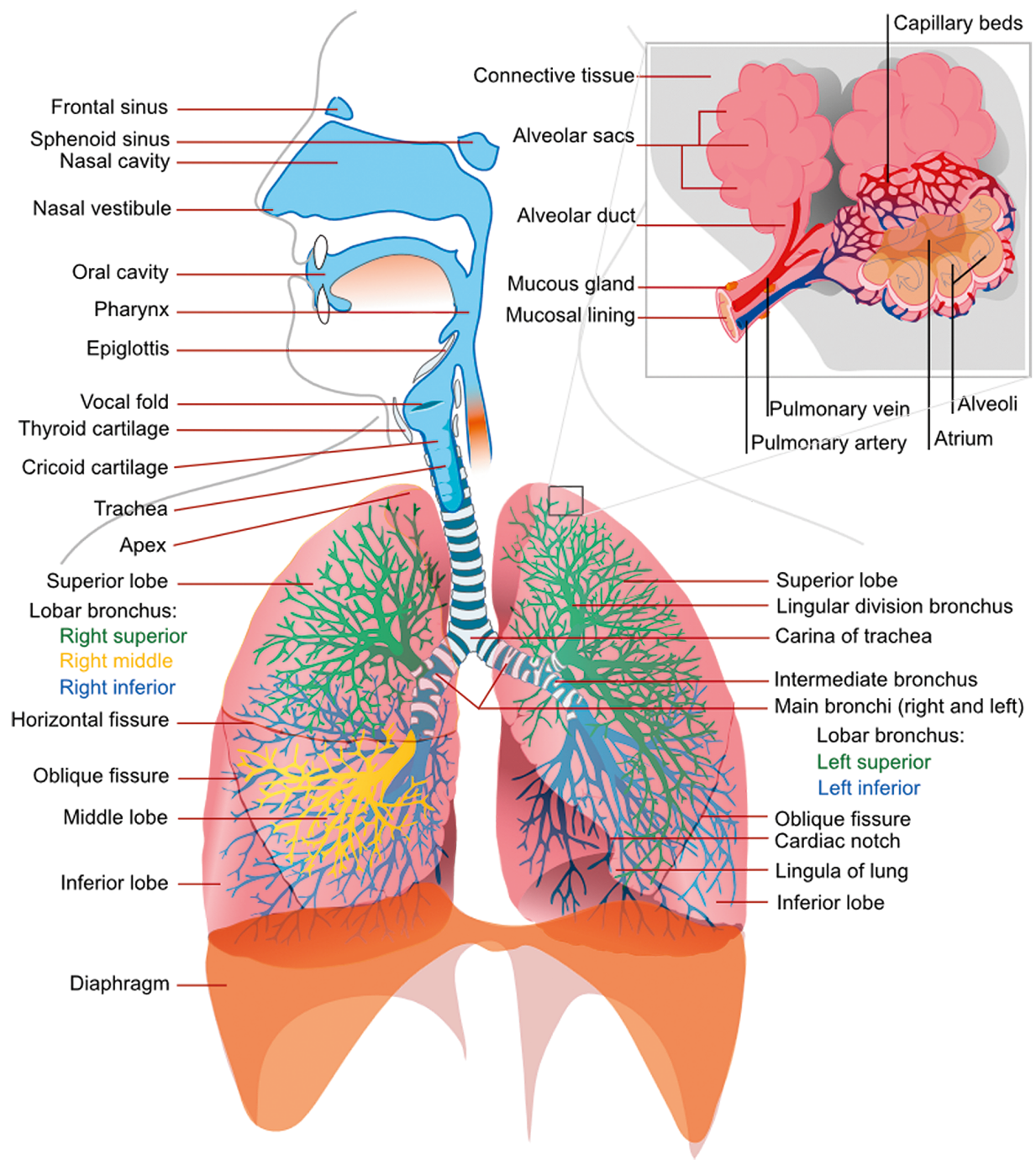

Figure 1. The respiratory system consists of the airways, the lung parenchyma, and the respiratory muscles that mediate the movement of air into and out of the body. The infolded image shows the alveoli and blood vessels (Re-printed from Wikimedia commons, LadyInHat, 2007).

\section{Lung diseases}

The major disease-induced cause of insufficient transfer of oxygen between alveoli and the capillary blood is due to mismatch of the air and blood in individual alveoli (Vander et al., 1994). To be most efficient, the right proportion of alveolar air flow (ventilation) and capillary blood flow (perfusion) should be available to each alveolus. Even in healthy persons there are factors, especially gravity, 
causing ventilation and perfusion mismatch in parts of the lung (Hedenstierna, 2000). In diseased persons with regional changes in lung compliance (stretchability), airway and vascular resistance can cause marked mismatch. The extremes are ventilated alveoli with no blood supply at all, or blood flowing through regions of the lung that have no ventilation. The mismatch can be more or less pronounced and yet quite significant (Vander et al., 1994). Examples of lung diseases with mismatched ventilation and perfusion are pneumonia, pulmonary embolism and chronic obstructive pulmonary disease (COPD) (Bajc et al., 2009a). Increased mismatch is correlated to disease advancement.

Emphysema is a type of COPD involving damage to the alveoli. In emphysema, the inner walls of the alveoli weaken and eventually rupture, creating one larger air space instead of many small ones. This reduces the surface area of the lungs and, in turn, the amount of oxygen that reaches the bloodstream. Furthermore, air becomes trapped in the damaged alveoli, leaving no room for fresh, oxygen-rich air to enter.

Heterogeneous ventilation has been demonstrated in lungs of allergic individuals, cigarette smokers, asthmatics and COPD patients (Altes et al., 2001; Emami et al., 2013; Jogi et al., 2011; Samee et al., 2003; Sovijarvi et al., 1982; Tgavalekos et al., 2007; Venegas et al., 2005). These subjects suffer from narrow and/or closed airways in various extents. Inflammation, secretions, and the shortening of muscle fibres around the bronchial walls, which obstructs airflow, cause narrowing of the airways. Asymptomatic smokers have been shown to have a more heterogeneous ventilation distribution compared to healthy non-smoking subjects (Emami et al., 2013). Narrowing of both larger and smaller airways can cause heterogeneities. It has been suggested that although larger asthmatic airways are dilated by inhalation of bronchodilator residual bronchial obstruction may still remain in some smaller airways, maintaining heterogeneous distribution (Sovijarvi et al., 1982). Furthermore, it has been concluded that the heterogeneous distribution of ventilation in asthma is an expression of the integrated system and not just the sum of independent responses of individual airways (Tgavalekos et al., 2007). Air trapping can be present even before pulmonary function tests are classified as abnormal or pulmonary symptoms become manifested (Ozer et al., 2005). Air trapping is for example due to aging, smoking and different obstructive diseases such as asthma (Ozer et al., 2005; Samee et al., 2003). The severity of air trapping has been shown to increase with age and smoking (Lee et al., 2000).

Advantageously, the lung has great compensation mechanisms, making it possible for the lung to reduce blood flow in volumes with low ventilation and to reduce ventilation to volumes with low blood flow. However, the compensation is nevertheless incomplete (Vander et al., 1994). 


\section{Diagnostic tests of lung function}

Several methods are used to get a good understanding of the function of the lung. Here follows a short description of the four most common methods.

\section{Lung function tests}

Lung function tests are the most common way of measuring lung function. A lung function examination can consist of dynamic flow rate and static lung volume measurements before and after inhalation of bronchodilator. Flow rates and volumes are measured via a mouthpiece while the subject inhales and exhales. An example of flow rate is the forced expiratory volume over one second $\left(\mathrm{FEV}_{1}\right)$. Different lung volumes are denoted forced vital capacity (FVC), vital capacity (VC), total lung capacity (TLC) and the residual volume (RV) (see Figure 2). An $\mathrm{FEV}_{1} / \mathrm{FVC}<0.7$ after bronchodilation is set to be the threshold value for persistent airflow limitation and thus of COPD according to the GOLD standard (GOLD-report, 2011). In combination with a ratio less than $0.7, \mathrm{FEV}_{1}$ is used for grading the severity of COPD from "mild" grade 1 to "very severe" grade 4 . RV and the ratio RV/TLC are related to indices of closed airways/air trapping. Closure of the airways by air trapping occurs when the small airways, the bronchioles, collapse and can be found using lung function tests in patients with emphysema and asthma as well as in lungs of elderly people (Gildea and McCarthy, 2010; Sharma and Goodwin, 2006).

The gas exchange across alveolar capillary membrane can also be measured by means of a single breath test of diffusion capacity for carbon monoxide (DLCO). The diffusion capacity and level of haemoglobin present in the blood are directly related. To exclude this dependence DLCO values are compensated for haemoglobin concentration (DLCOc) (Gildea and McCarthy, 2010). DLCOc is a measure partly related to supposed ventilation/perfusion mismatch. In addition DLCOc is also related to the properties of the lung parenchyma that separates the alveolar gas from the red blood cells.

The total cross section of the tubes increases dramatically down the respiratory zone which results in a very low air flow resistance. A consequence of this is that a doubling of the air flow resistance, due to some disorder, would hardly be identified using common lung function tests (Bake, 2000). Furthermore, the outcome of a lung function test is a summation of the status of the whole lung, normal and/or abnormal volumes. Therefore, significant changes in lung function may occur before a deviation from the normal can be identified.

When individual values are compared to normal values for the same gender, age and height, the values are referred to as percentage of predicted normal $(\%$ pred.). The percentage of predicted normal is used to grade the severity of the abnormality. 


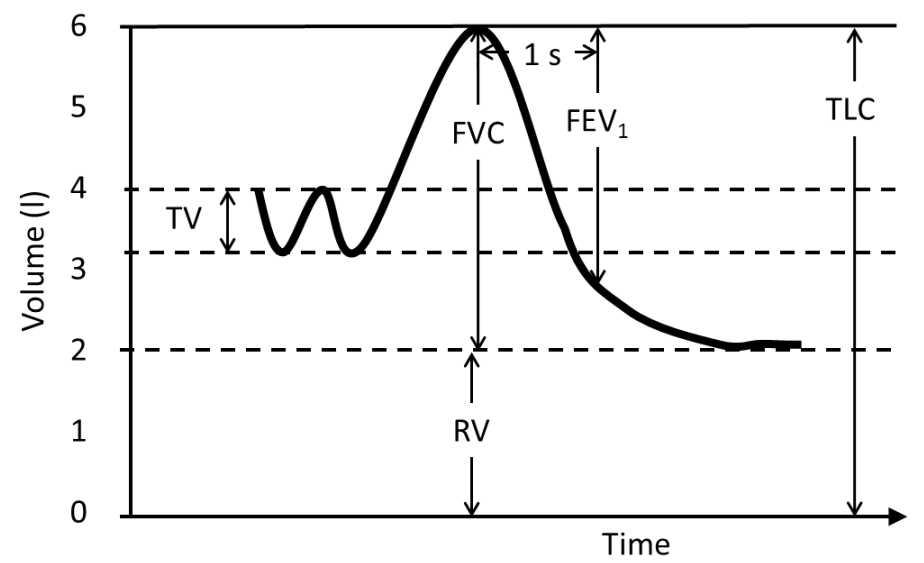

Figure 2. A schematic spirogram with tidal respiration, followed by maximal exhalation to residual volume. The exhalation is rapid for the forced expiratory volume measured over one second $\left(F E V_{I}\right)$. Remaining measured volumes used in this thesis are the resting tidal volume (TV), the residual volume (RV), forced vital capacity (FVC) and the total lung capacity (TLC). After (Gildea and McCarthy, 2010).

\section{High resolution computed tomography, HRCT}

Images from inspiratory and post-expiratory high resolution computer tomography (HRCT) are used to verify emphysema in patients. HRCT images have shown air trapping in patients with obstructive lung disease (Chen et al., 1998), moderate asthma (Laurent et al., 2000) and smokers (Spaggiari et al., 2005).

\section{Planar scintigraphy}

Planar scintigraphy results in two dimensional images of the deposited radioactive isotope either for ventilation of perfusion studies. Images are acquired from different angles around the subject. The most common clinical indication for lung scintigraphy is to determine the likelihood of pulmonary embolism (Parker et al., 2012). Less common indications are for example to document the degree of resolution of pulmonary embolism, quantify differential pulmonary function before pulmonary surgery for lung cancer or to evaluate lung transplants (Parker et al., 2012). Evaluation of the images is often qualitative.

\section{Single photon emission computed tomography, SPECT}

Single-photon emission computed tomography, SPECT, is increasingly used in respiratory research and in clinical applications as a replacement for planar scintigraphy. The resulting SPECT images show the activity distribution in three dimensions. Therefore, the deposition of activity is not superimposed as is the case of planar scintigraphy, which is beneficial. The same indications hold for SPECT 
as for planar scintigraphy. The evaluation of the SPECT images can be qualitative or quantitative.

Most common in the clinic has been to use qualitative evaluation searching for pulmonary embolism (Bajc et al., 2009a, b). Additional diagnostic yield from such a pulmonary embolism SPECT examination includes COPD, heart failure and pneumonia (Bajc et al., 2009a). Quantitative lung SPECT has been shown useful for assessment of for example regional severity of emphysema (Nagao et al., 2000; Suga et al., 2010), aerosol deposition and clearance (Eberl et al., 2006), ventilation and perfusion ratios (Suga et al., 2010; Sando et al., 1997), early radiation-induced lung injury (Zhang et al., 2012), regional perfusion and ventilation (Petersson et al., 2006; Ax et al., 2013) and of ventilation heterogeneity (paper II and IV).

\section{Radiotracers for lung SPECT}

The most common radioactive isotope for lung SPECT is technetium $99\left({ }^{99 \mathrm{~m}} \mathrm{Tc}\right)$, produced using a Technetium generator. The isotope emits photons with $140 \mathrm{keV}$ $(89 \%)$ with a half value layer of $4.3 \mathrm{~cm}$ in soft tissue (ICRU-44, 1989) and with a half-life of 6 hours (Mougeot et al., 2012). ${ }^{99 \mathrm{~m}} \mathrm{Tc}$ is an almost pure gamma emitter with sufficient energy to escape the body and with a half-life practical for diagnostic imaging.

Common for ventilation examinations is to use Technegas (Burch et al., 1986) which is ${ }^{99 \mathrm{~m}} \mathrm{Tc}$ labelled carbon filled nano-particles. The gas is produced using the Technegas Generator (Tetley Manufacturing Ltd., Sydney, Australia) by adding ${ }^{99 \mathrm{~m}} \mathrm{Tc}$ to the graphite crucible and thereafter vaporise it at $2500^{\circ} \mathrm{C}$ in an $100 \%$ argon atmosphere. The gas is inhaled (left image in Figure 3). Burch et al. (Burch et al., 1986) reported that the diameter of the particles is $5 \mathrm{~nm}$. However, more recently Harris and Harris have suggested that the diameter is between 10 and $100 \mathrm{~nm}$ (Harris and Harris, 2001). The particle size allows good peripheral penetration with little central deposition and the agent has prolonged pulmonary retention, clearing from the lungs with the half-life of the radionuclide (Monaghan et al., 1991). The distribution of the carbon particles in lungs is like the earlier much used noble inert gas ${ }^{133} \mathrm{Xe}$ (Xenon) (Amis et al., 1990), which offered homogenous distribution throughout the lungs (Van Beek, 2004). A stable deposition in the lung makes Technegas suitable for tomography. However, $\mathrm{Xu}$ et al. (Xu et al., 2001b) demonstrated an initial phase of rapidly reducing number of counts thought due to clearance of Pertechnegas. The image becomes stable first approximately $50 \mathrm{~min}$ after inhalation. Pertechnegas is produced if oxygen is present in the argon gas when producing Technegas (Monaghan et al., 1991). Technegas was used in paper II and IV. Another commonly used radiopharmaceutical is the ${ }^{99 \mathrm{~m}} \mathrm{Tc}$-diethylenetriaminepentaacetic acid (DTPA) with a less uniform distribution in the lungs compared to Technegas (Parker et al., 2012). ${ }^{81 \mathrm{~m}} \mathrm{Kr}$ (Krypton) is also used for ventilation examinations. 
The radiopharmaceutical used for perfusion imaging is macro aggregated albumin (MAA) connected to ${ }^{99 \mathrm{~m}} \mathrm{Tc},{ }^{99 \mathrm{~m}} \mathrm{Tc}-\mathrm{MAA}$. The biologic half-life of the MAA in the lungs varies (usually 1.5-3 h) (Parker et al., 2012). MAA- ${ }^{99 \mathrm{~m}}$ Tc is injected intravenously in the hand or arm. The MAA are trapped in the capillary net in the alveoli walls at the first passage. Since the MAA particles temporary prevent the blood flow in the vessels they are trapped in, the number of MAA particles must be limited. At the same time the number of MAA particles has to be large enough to generate a representative image of the perfusion distribution.

\section{The gamma camera}

Gamma camera images (projections) can be generated by using human subjects together with a radioactive tracer and a gamma camera (paper II and IV). The inhalation of Technegas can be performed as shown in Figure 3a. Also shown is a subject during acquisition of gamma data positioned in-between the two gamma camera heads (Figure 3b).
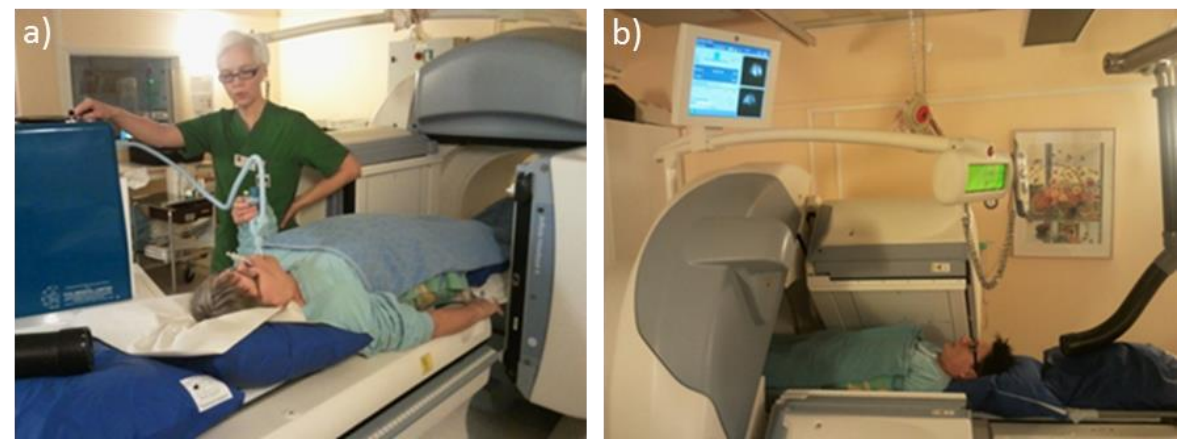

Figure 3. Acquisition of gamma data of two healthy volunteers. a) A subject inhales the radioactive gas through a mouthpiece. A nose clip prevents the inhaled gas to escape out through the nose. b) A subject is positioned in between the two gamma camera detectors. The radiation from the gas is collected at 120 different angles, equally spaced, over $360^{\circ}$. The collection time is about 20 minutes.

A gamma camera commonly has one to three heads. The more heads the more counts can be collected per time unit. A scintillator detector head consists of a guard, a collimator, a flat scintillating detector crystal, a light pipe, an array of photo multiplier (PM) tubes completely covering one side of the light pipe surfaces, and electronics (Figure 4a). The guard protects the collimator from being damaged. The collimator restricts the photons generating the image so that the image can be directly interpreted as the spatial (geometric) distribution of the emitting isotope. Collimators are generally manufactured in lead and in honeycomb shape. The holes can be parallel, diverging or converging. The collimator 
can also be a single thin opening, a so called pin-hole collimator. Collimators have different thicknesses of the walls (septa) depending on the energy of the emitted photons. Typical size of holes is between 1.5 and $2 \mathrm{~mm}$ for low energy photons. Two commonly used ${ }^{99 \mathrm{~m}} \mathrm{Tc}$ collimators are the low-energy high resolution (LEHR) and low-energy general purpose (LEGP) collimators. The smaller and longer the holes are the higher resolution in the image (Figure 5b). At the same time small and long holes lower the number of registered photons. The most widely used scintillator crystal is $\mathrm{NaI}$ (sodium iodide) converting the incident radiation energy to promt (direct) fluorescence, i.e. emission of visible light (Knoll, 1989). The thicker crystal the larger proportion of the emitted light will be included in the resulting photo peak. A thicker crystal will also degrade the spatial resolution. The visible radiation is led through the light pipe (guide) to the photocathode in a PM tube. The photocathode absorbs the light and converts it to low-energy electrons. The subsequent multiplication of those electrons in the PM tube results in electrical pulses corresponding to the $\mathrm{x}$ and $\mathrm{y}$-coordinates of the photon-light event and the energy of the photon. Coordinates corresponding to photons having their energy in the accepted interval are visualised by a dot (a count) in an x,y-coordinates system. After sufficient number of photons detected, an image is visible (Figure 5a). Performing a SPECT acquisition the radiation is collected at a large number of different angles around the subject, typically equally spaced, over $360^{\circ}$. Auto-contouring or a constant distance to the detector can be employed.

On the gamma camera an X-ray source and a detector can be mounted. A low-dose CT examination can be performed and used for attenuation compensation and for localisation, i.e. delineation of imaged organ and fused with the SPECT image.

\section{Physical effects}

Photons emitted from the radioactive isotope are scattered, attenuated (absorbed) in the body or escape without interaction (Figure 4a). Photons escaping without interaction are called primary photons. Photons headed towards the collimator either pass through the collimator or are attenuated in the collimator septa depending on their incoming angle. Some photons scattered in the body also pass the collimator. The scattered photons have lower energies and most of them are discriminated in a later signal-processing step using an energy window technique (Figure 5b). 
a)

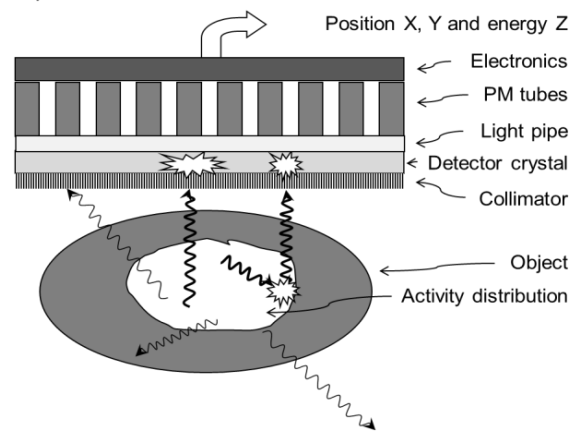

b)

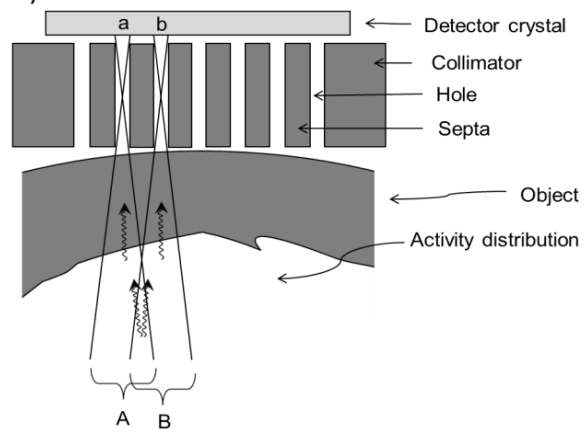

Figure 4. Shown in a) is a schematic image of a section through a subject or phantom and a detector head with a parallel hole collimator. Wavy arrows indicate photon directions. A photon is absorbed in the collimator, one detected in the crystal, one scattered and then detected, one escaping without detection and one absorbed in the object. An expanded view is shown in b) to illustrate the relationship between the diameter of the hole and the spatial resolution of the image. Photons originating from sector $A$ with an appropriate angle will be detected in the crystal at $a$, and photons from $B$ in $b . O b$ serve the overlap of the two sectors.
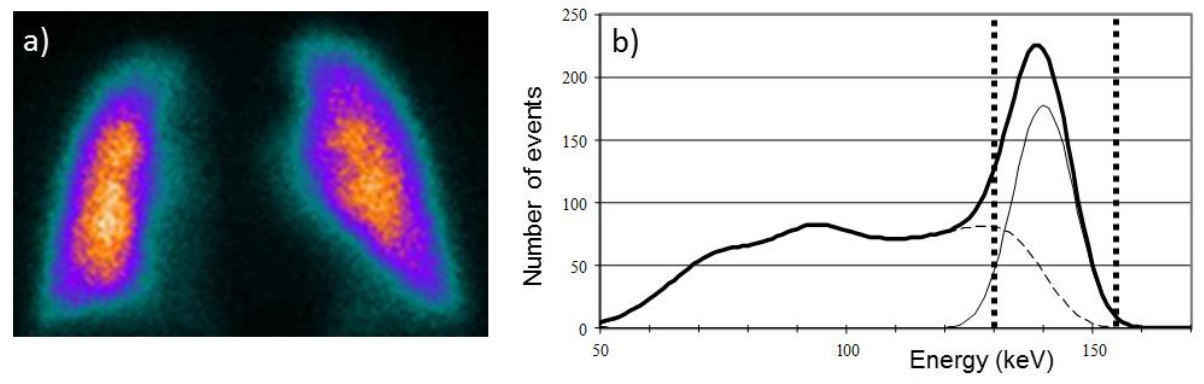

Figure 5. Shown in a) is a lung function projection taken from the front of a healthy volunteer, visualised using a colour scale. Yellow corresponds to a high level of activity and green to a low level. Shown in b) is a typical histogram of ${ }^{99 m}$ Tc photons detected by a gamma camera showing their energy and relative number. The primary photon peak (thin line) and scattered photons (thin dashed line) together create the total distribution (thick line). The interval of accepted energies (dotted lines) is set over the peak of the distribution in which most of the primary photons and some of the scattered photons have their energies. 
Attenuation, scatter and the distance between the deposition and the detector are effects that alter the information in the resulting projection. Attenuation of photons in the imaged object results in a reduced number of detected counts in the projections. A scattered photon, accepted by the energy window, originally from a deposited ${ }^{99 \mathrm{~m}} \mathrm{Tc}$ particle in one position will contribute to the apparent deposition in another position. Therefore scattered photons result in a shadowlike image added on top of the primary image. Furthermore, the spatial resolution degrades with increasing distance from the collimator surface, i.e. a particle deposition positioned far away from the collimator will appear wider in the projection compared to a close deposition. This effect is called the collimator detector response (CDR). Finally, due to the limited amount of administered activity and acquisition time the projections are more or less noisy.

\section{Acquisition parameters}

The amount of administered radioactivity to human subjects is limited to be as low as reasonably achievable according to the ALARA principle since ionising radiation can induce cancer and heritable disease (ICRP-103, 2007). The risk associated with ionising radiation is estimated using the quantity effective dose. The effective dose to an average Swedish person is estimated to $3 \mathrm{mSv}$ per year including contributions from medical examinations and radon (Strålsäkerhetsmyndigheten, 2011). Another limitation is the total acquisition time that must be reasonable and adapted to how long time a person can lie still without moving.

Important acquisition parameters for a given detector are activity level, radius of rotation, collimator, energy window, number of projections and acquisition time per projection. Together with the distribution of the activity in the lung of a human subject these parameters determine the number of counts, noise level, spatial resolution and contrast in the projections.

\section{Monte Carlo simulations and phantoms}

Gamma camera images can also be generated using virtual anthropomorphic phantoms with various activity distributions together with Monte Carlo simulations (paper I, II and III). Using a virtual phantom instead of humans is appropriate when evaluating the outcome of various activity distributions in a human surrounding. This method enables control over all involved parameters. Various acquisition, reconstruction and image analysis strategies can be evaluated without the use of radioactivity, clinical equipment or the need of human subjects.

Virtual phantoms can be voxel-based or nurbs-based. A voxel-based phantom is static and the extent of organs, tissues and outline of the body are difficult to manipulate. Voxman (Figure 6a), is one example, based on a CT-study of a 
man (Zubal and Harrel, 1990; Zubal et al., 1994) (paper I). Various activity distributions can be applied to this phantom. The newer generation of four dimensional nurbs-based phantoms are more flexible. This since different sizes and shapes of different tissues can be selected and natural movements caused by heartbeat and respiration can be modelled. The NCAT software (Segars and Tsui, 2001) generates such phantoms (Figure 6b) (paper II and III). Various activity distributions can also be applied to this phantom.
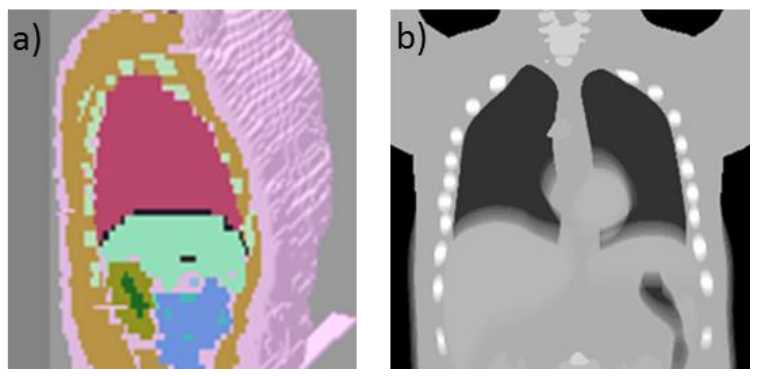

Figure 6. Shown are thorax cross-sections through phantoms. a) A sagittal crosssection of the static Voxman phantom where each colour corresponds to a specific tissue. The lung is red (image obtained from (Zubal et al., 1994)).b) A cross-section of the NCAT phantom visualising selected tissues, respiratory motion and heartbeat.

The projections from the activity distributions are simulated using Monte Carlo techniques. The SIMIND software (Ljungberg and Strand, 1989) is such a Monte Carlo code. The technique is a statistical method, based on random numbers, simulating the photons' paths through specified phantom and gamma camera geometries. A large number of emitted photons are followed from the position of the radioactive particle inside the phantom through organs and tissues, and collimator, to the detector crystal where they are absorbed. The probability of attenuation and scatter is determined by the cross section of the specified materials. The thickness of the crystal and the specified energy discrimination window is also taken into account when the final projection is created. Simulations can be made for various gamma camera systems and phantoms, e.g. various isotopes, activity distributions, collimators, crystal thicknesses, energy windows, fixed radius of rotation or contouring, number of projections, pixel resolutions and pixel sizes. The simulated projections have the same energy and spatial resolution as projections of the defined system.

The number of photons to be simulated has to be decided depending on which activity levels to be studied. A strategy can be to simulate projections with very low noise levels (with a coefficient of variation, $\mathrm{CV}$, of approximately $0.5 \%$ as in paper II and $2 \%$ in paper I) by simulating a large number of photons. Then the low-noise projections is normalised to a total number of counts representative 
to that of clinical studies. Thereafter, the number of counts in individual pixel elements in the projections is replaced by random deviates drawn from a Poisson distribution that had the number of counts in the pixel as the mean value. In this way it is possible to adjust the projections to correspond to various activity levels and to generate Poisson noise typical of realistic projections. As an example, the $\mathrm{CV}$ in projections from a uniform activity distribution $(\mathrm{CV}=0 \%)$ after such procedure was approximately $15 \%$ (paper II and III).

Using this strategy, a number of noise realisations are easily made, corresponding to repeated gamma camera acquisitions of the exact same activity level and distribution. The use of noise realisations makes it possible to analyse the variability in measured uptake due to statistical noise and to calculate average values of uptake.

\section{Reconstruction techniques}

The two-dimensional projections acquired at different angles around the object are reconstructed into a three-dimensional image of the activity distribution of the studied organ. Typical reconstruction algorithms are the traditional filtered back-projection (FBP) (Rosenfeld and Kak, 1982) and the iterative ordered subsets expectation maximisation (OSEM) (Hudson and Larkin, 1994). The degrading effects such as attenuation, scatter and collimator detector response (CDR), affect the quantitative accuracy and image quality of the reconstructed images. Therefore, methods reducing these effects are frequently applied. Since the reconstructed image often is excessively noisy, reduction of the noise is also necessary.

\section{Filtered back projection, FBP}

FBP is described in detail by Rosenfeld and Kak (Rosenfeld and Kak, 1982). The reconstruction method is analytical and computationally fast. Attenuation and scatter compensation can be applied to the projections before reconstruction. Non-uniform attenuation compensation can be performed using the CT scan of the subject or a map of the phantom. Images reconstructed using FBP has streak artefacts due to limited number of projections, and the adherent ramp filter amplifies the noise in the image (Figure 7a).

\section{Ordered subset expectation maximisation, OSEM}

Thanks to the capacity of modern computers, iterative reconstruction algorithms are today operational in a clinical environment. A common statistical iterative reconstruction algorithm is the maximum likelihood expectation maximisation (MLEM) that maximises a likelihood function (Shepp and Vardi, 1982). The MLEM iteration works in two steps where the first step is to determine the expected projections based on the current estimate of the activity distribution (for- 
ward projection). The second step (back projection) uses the ratio between the estimated and measured projections to adjust the current activity distribution to be closer to the most likely solution where likelihood is maximized. This means that for each iteration the reconstructed image takes a step towards the statistically most likely activity distribution that could generate the acquired projections. This algorithm is known to be effective but slow. In the faster OSEM algorithm the projections are divided into subsets and only a subset at a time is used for each update. The reconstructed image obtained from one MLEM iteration is approximately the same as from one OSEM update so the OSEM algorithm is speeded up by a factor equal to the number of subsets. The number of OSEM updates is equal to the iteration number times the number of subsets. For higher numbers of iterations the similarity between the forward projected projections and the acquired projections still increases while the reconstructed image gets noisier.

Iterative reconstruction methods have the inherent potential to compensate for physical effects such as attenuation, scatter and the collimator detector response. This since OSEM can make use of a probability matrix describing the emission and detection process in the body and detector. Attenuation is compensated for using a density map of the phantom or the subject (CT-scan). Scatter compensation can be performed using the effective source scatter estimation (ESSE) (Frey and Tsui, 1996; Larsson et al., 2010). Compensation for CDR can be performed using an analytic geometrical model.

\section{Noise reduction}

The noisy reconstructions can be post-filtered with for example threedimensional Hann- and Butterworth filters. The filters suppress the high frequencies in the image to various extents. The shape and therefore the effect of the Hann filter depends on the cut-off frequency of the filter function while that of the Butterworth filter depends on both cut-off frequency and power (RJ Ott, 1988).

\section{Reconstruction parameters}

Important factors for the reconstructed image are the use of compensation methods for the physical effects, the reconstruction parameters such as the number of updates for OSEM and, the cut-off frequency and power of the noise reduction filter. Together with the acquisition parameters the reconstruction parameters determine the noise level, spatial resolution and contrast in the final image. 


\section{The resulting image}

\section{Phantom images}

The characteristic streak artefacts of a FBP reconstructed image are visualised in Figure 7a, which are not found in the OSEM reconstructed image in Figure $7 \mathrm{~b}$. Both these reconstructions are based on a uniform activity distribution in a phantom lung but since we limit the amount of activity and acquisition time the reconstructed images become patchy. Furthermore, the reconstructed activity distribution at the periphery of the lung is blurred. This is due to the limited spatial resolution of the SPECT system of about 1-1.5 cm (for a LEHR collimator, expressed in full width at half maximum, FWHM). This periphery effect is most easily observed in the OSEM reconstruction in Figure $7 \mathrm{~b}$.

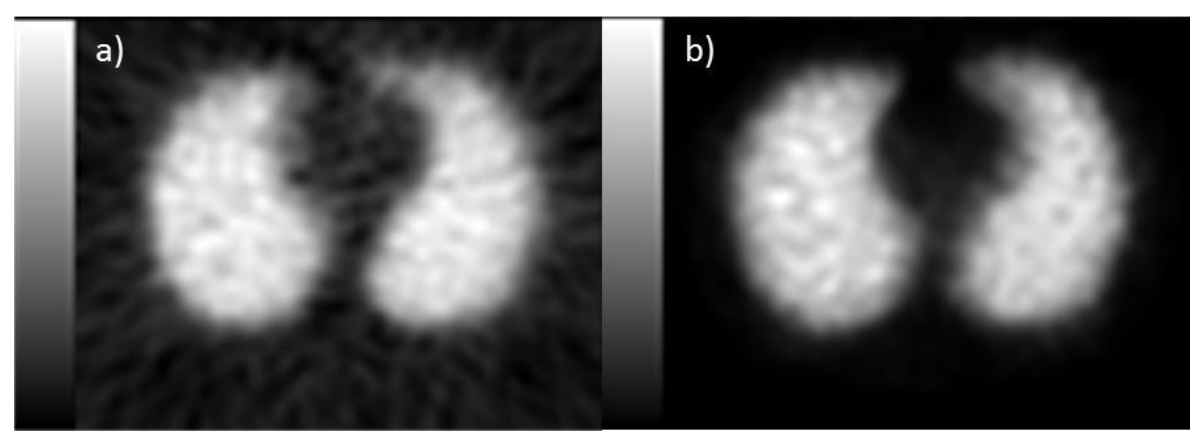

Figure 7. Transversal reconstructed slices based on a uniform activity distribution in a phantom lung. The CV level inside the lung of both slices is about 5\%. a) The slice was reconstructed using FBP, an LEGP collimator and a cut-off frequency of the Hann filter of $1.2 \mathrm{~cm}^{-1}$. b) The slice was reconstructed using OSEM with 60 updates with the same collimator and similar cut-off frequency. Notice the streak artefacts of FBP reconstructions, due to the limited number of projections that are not visible in the OSEM reconstruction. The slices originate from work I.

\section{Human images}

In lung ventilation SPECT images of a healthy human subject the distribution will not be as uniform as the simulated uniform activity distribution, an effect assumed to be related to the shape of the bronchial tree and the gravity influencing the lung (Figure 8a, b). In a diseased subject, loss of Technegas particle deposition is expected in parts with reduced regional ventilation (Figure 8c). Furthermore, among diseased subjects it is not unusual to find hotspots in the ventilation SPECT images (Figure 8c). These hotspots may be caused by obstruction of central airways (Pellegrino et al., 2001). During expiration the Technegas facilitates airflow limitations as pronounced and oscillating narrowing of the air- 
way walls. This airflow limitation causes strong turbulence resulting in high impaction of Technegas particles (Pellegrino et al., 2001). In less diseased subjects such pronounced hotspots are less likely.
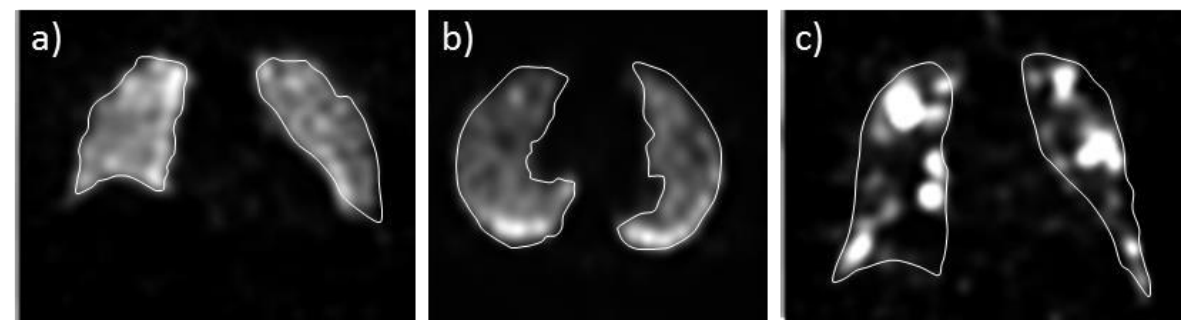

Figure 8. OSEM reconstructed slices of Technegas distribution in two healthy volunteer $(a, b)$ and a COPD patient (c). Lung contours from corresponding CT slices are outlined. Inhalation and acquisition was performed with the subjects in supine position and therefore the influence of gravity can be observed in the transversal slice (b) and not in the coronal slices $(a, c)$. The patient' distribution is very patchy with loss of particle deposition next to bright hotspots while the healthy subject's distribution is more uniform (from paper II).

\section{Optimisation}

The choice of acquisition and reconstruction parameter values is very important. Optimal values have to be found in relation to the image and the image diagnostic task.

Guidance in the choice of parameter values can be given using trade-off plots (Olsson et al., 2007; Turkington et al., 2007) of image quality such as contrast, resolution and noise in simulated images (paper I). The choice of parameter values is depending on what noise level is acceptable in the image. For visual interpretation of the final image, visual grading experiments can be used to determine the optimal parameter values.

For quantitative assessment the quantitative method itself can be used for optimising the parameter values (paper III). With the use of appropriate activity distributions in phantoms, Monte Carlo simulations, reconstructions and image analysis, various parameter values can be tested. The most beneficial combination can then be identified. 



\section{IMAGE AND COMPUTER PROCESSING}

The NCAT software (Segars and Tsui, 2001) was used in paper II and III for creation of dynamic lung phantoms and activity distributions.

The SIMIND software (Ljungberg and Strand, 1989) was used in paper I, II and III for Monte Carlo simulations of gamma camera projections.

OSEM reconstruction including attenuation compensation was performed on an eNTEGRA workstation (General Electric) in paper I. OSEM reconstruction software including attenuation, scatter and CDR compensation developed at Johns Hopkins University (Baltimore, MD, USA) was used in paper II, III and IV.

All other computer processing was performed using in-house software developed in Interactive Data Language (IDL; ITT Visual Information Solutions, Boulder, CO, USA). Examples of processing are: Poisson noise handling, FBP including attenuation compensation, post-filtering, lung segmentation, calculations of $\mathrm{AUC}\left(\mathrm{CV}>\mathrm{CV}_{\mathrm{T}}\right)$ values, evaluations of the CVT method, optimisation of acquisition, reconstruction and analysis parameter values. Agnetha Gustafsson developed the code for FBP including attenuation compensation while the remaining code was developed by me. 



\section{EVALUATION OF RECONSTRUCTION ALGORITHMS}

At that time, two algorithms were available for clinical use in commercial systems, namely FBP and OSEM. The comparison was made using trade-off plots (Olsson et al., 2007; Turkington et al., 2007) of image quality parameters such as contrast, noise and resolution. The parameters studied were the number of iterations and subsets for OSEM, collimator, noise-reduction filter and activity level. Paper I.

\section{Material and Methods}

In this study the static Voxman phantom (Zubal et al., 1994) was used with a homogenous activity distribution throughout the lung, and a distribution including lines and spherical object (Figure 9). The spheres had a 50\% lower activity level compared to the uniform surrounding material, while the lines had a much higher activity level. The lines were 1-voxel wide, extending in a cranial-caudal direction from end to end of the lung, and the spheres had a diameter of $23.1 \mathrm{~mm}$. The homogeneous distribution made it possible to measure the resulting noise in the reconstructions in a large number of voxels. In the object distribution, the resolution could be measured without being disturbed but yet in a complex surrounding.
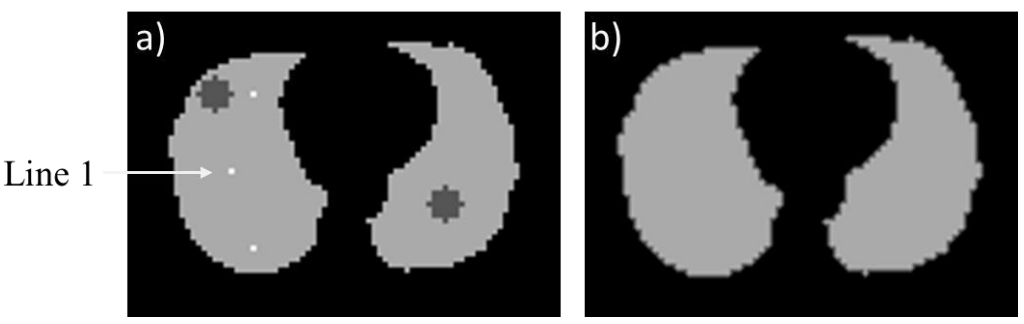

Figure 9. Transversal slices through the a) object distribution with homogeneous activity concentration in the lungs on which are superposed three lines with high activity and two spheres with low activity. b) A homogeneous distribution with a uniform activity concentration throughout the lungs.

The projections were simulated using the SIMIND software. Simulations were made for a VG Millennium gamma camera (15.9mm NaI detector), equipped with a LEGP or a LEHR collimator (GE Healthcare, USA).

Three count levels were considered for the LEGP collimator. The total numbers of counts for the projections was $5 \times 10^{6}, 11 \times 10^{6}$ and $22 \times 10^{6}$, respectively 
(count level 1,2, and 3). The LEHR projections were normalized to a total number of counts of $7 \times 10^{6}$ (count level $2 \mathrm{HR}$ ). The reduced number of counts in count level 2HR compared to count level 2 is due to the lower sensitivity of the LEHR collimator compared to the LEGP collimator.

The projections were reconstructed using both FBP and OSEM. OSEM reconstruction was made using 4 to 90 updates. Attenuation compensation with the same density distributions were performed for both reconstruction techniques. Scatter compensation was not used. This was since a pre-study, comparing reconstruction with attenuation compensation, with or without scatter compensation, showed unchanged spatial resolution and just a small increase in noise. The influence on image quality using CDR was not investigated since CDR was not feasible on the eNTEGRA workstation where the OSEM reconstructions were performed.

The noisy reconstructions were post-filtered with three-dimensional Hann or Butterworth filters. The different parameters investigated for Hann filters were 13 cut-off frequency values in the interval $0.7-1.9 \mathrm{~cm}^{-1}$; and for Butterworth filters were 18 combinations of cut-off frequency values from 0.3 to $0.8 \mathrm{~cm}^{-1}$ and power values of 5,7 and 10 .

The noise was measured as CV (coefficient of variation) inside the lungs of the homogeneous distribution after eroding a seven-voxel margin at the periphery (Figure $10 \mathrm{a}, \mathrm{b}$ ). This margin was excluded to avoid the periphery effect.

Spatial resolution was determined by measuring the full width at half maximum (FWHM) of the line spread function, using linear interpolation (Figure 10 $\mathrm{c}, \mathrm{d})$. The measurements were done in three equally spaced transverse slices in both the coronal and the sagittal directions over line 1 and the mean value was reported. Reconstructions from the object phantom contained a background level that had to be subtracted before the FWHM could be measured.

Contrast was calculated as the relative difference between the low count voxel values in the left lung sphere, and the surrounding voxel values (Figure 10 e, f). The contrast was estimated in reconstructions using projections without noise. This was because a pre-study on data with noise had shown, that the mean value of the contrast for a number of noise realisations increased with increasing numbers of iterations in a similar way as without noise. The mean value also reached the same constant contrast level as for noise-free reconstructions. The reconstructions based on noisy projections were not post-filtered.

The comparison between OSEM and FBP was based on studies of the relationship between statistical noise and spatial resolution for the different cut-off frequencies of the noise reduction filter. 

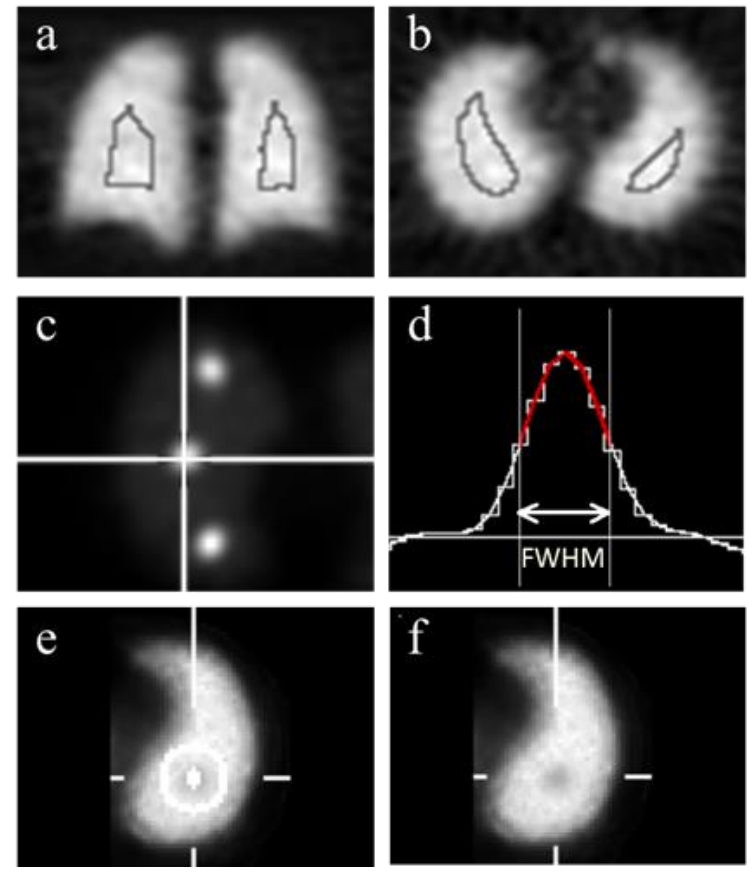

Figure 10. Illustrations of volumes and profile used for noise, resolution and contrast measurements. a) A coronal and b) a transversal slice through a FBP reconstruction based on projections from the homogeneous distribution is. The volume of interest is outlined. c) A transversal slice through the right lung of an OSEM reconstruction, based on projections from the object distribution, showing the coronal and sagittal positions of the profile over line 1. d) A profile over line 1 showing FWHM. e) A transversal slice through the left lung of an OSEM reconstruction, based on projections from the object distribution, with the volumes of interest of the low activity region and the surroundings highlighted and f) without.

\section{Results and discussion}

The study showed that OSEM is the preferred reconstruction method since OSEM obtains higher resolution at a lower noise level compared to FBP. A large number of iterations is needed, the LEHR is preferable, and the cut-off frequency is essential but the number of subsets and type of filter are not.

The evaluation of the number of iterations used in OSEM was based on the image quality parameter contrast. After 60 updates the contrast value began to reach a plateau and that number of iterations was therefore considered to be sufficient (Figure 11). To shorten calculation time and keep image quality constant, 6 subsets were used with 10 iterations.

The objective is to obtain high resolution in combination with a low noise level. For higher activity levels, a cut-off frequency could be chosen resulting in a lower CV and FWHM than at lower activity levels (Figure 12a). A LEHR col- 
limator generated a better combination of CV and FWHM than a LEGP collimator, for a constant activity level (Figure 12a). For a constant activity level, OSEM was to preferred over FBP for both the LEGP and the LEHR collimators. OSEM with the LEHR collimator could generate the highest resolution together with the lowest noise level (Figure 12b).

The best choice of filter is dependent on CV level. Generally Hann showed the lowest FWHM values for CV levels under 4-5\% and Butterworth power 10 showed lowest FWHM values above this CV level. The differences in FWHM between these filters were quite small, less than $0.5 \mathrm{~mm}$, for CV levels above $3 \%$ using OSEM.

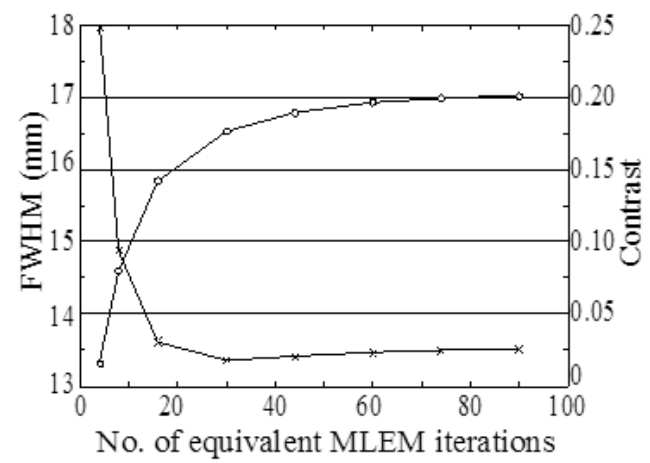

Figure 11. Resolution ( $x$ ) and contrast (o) versus numbers of updates (equivalent iterations using OSEM) with two subsets, for count level 2 and LEGP collimator, without statistical noise or filters. The numbers of updates ranged from 4 to 90.
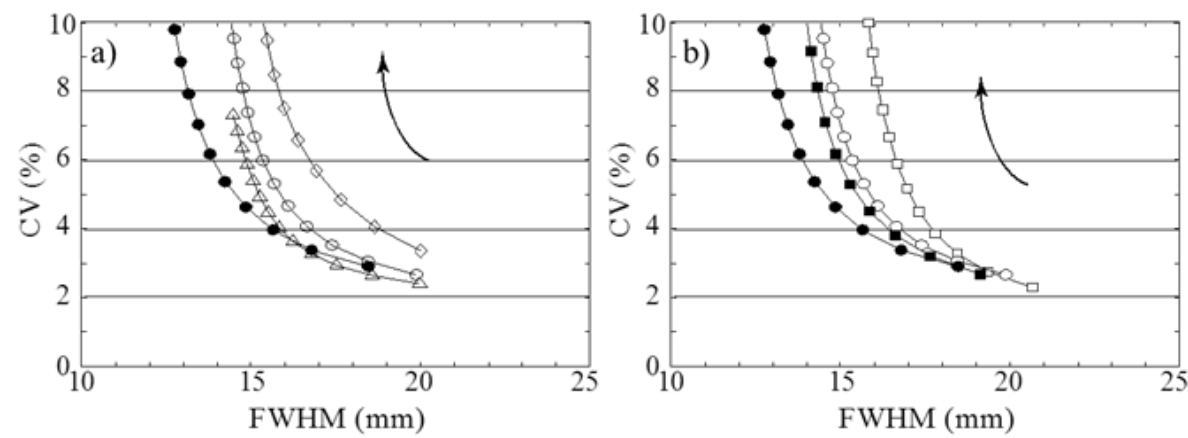

Figure 12. Trade-off plots of noise versus resolution. a) OSEM, using 60 updates for count level 1 using LEGP collimator $(\diamond)$, count level 2 using LEGP collimator (O), count level 3 using LEGP collimator $(\triangle)$ and count level $2 H R$ using LEHR collimator (•). b) Count level 2 for LEGP collimator (open symbols), and count-level $2 H R$ (110 $M B q$ ) for LEHR collimator (filled symbols), using OSEM with 60 updates (O) and FBP (). The arrows show increasing cut-off frequencies in steps of $0.1 \mathrm{~cm}^{-1}$ with Hann filters, starting with $F=0.7 \mathrm{~cm}^{-1}$. 


\section{DEVELOPMENT AND OPTIMISATION OF THE CV T-METHOD}

\section{The coefficient of variation}

In this thesis heterogeneity inside the lung in the SPECT image is measured using the coefficient of variance, $\mathrm{CV}$, i.e. the standard deviation divided by the mean value. The $\mathrm{CV}$ value will be low in homogenous parts of a distribution and higher in heterogeneous parts, i.e. where there are gradients. CV will also be lower in homogeneous parts with a high mean value compared to homogenous parts with a lower mean value. To illustrate the behaviour of these values, CV, the mean and the standard deviation, were calculated for a kernel (cubic volume) that stepped through a fictive activity distribution (Figure 13). For each step the values were derived based on the voxels enclosed by the kernel. The activity distribution consisted of large and small spheres (relative to the kernel size) with low means, very low means and high levels compared to the surrounding and, with Poisson noise incorporated. The surrounding level corresponds to "healthy" deposition of activity, spheres with low levels to "unhealthy" loss of activity and high levels spheres to "unhealthy" hotspots. Both reduced and increased particle deposition will generate increased $\mathrm{CV}$ values compared to the surrounding, but the highest CV values will be generated for a large loss of activity. Notice that the lowest CV value is found in the "healthy" surrounding while the mean and the standard deviation both have higher and lower values for kernel positions including spheres. This indicates that the $\mathrm{CV}$ is a better measure than the mean and standard deviation.

\section{The quantitative $\mathrm{CV}_{\mathrm{T}}$-method}

The tree-dimensional reconstruction was subjected to a, for example $3 \times 3 \times 3$ $\mathrm{cm}^{3}$ (i.e. $9 \times 9 \times 9$ voxels), cubic kernel that stepped through the volume. A step was a one-voxel-step in the $\mathrm{x}, \mathrm{y}$ or $\mathrm{z}$ direction. In each step, a CV value was calculated. The kernel defines which voxels of the reconstruction the CV calculations were based on. Voxels outside the defined lung volume were excluded from the CV calculation. The kernel approach used does not exclude any volume of the lung in the analysis. The calculated CV values were stored in a new matrix, the CV matrix, creating a parametric image (see section "Parametric image and sub-volumes", page 54-56). All the calculated CV values for each reconstruction were constructed and plotted as a normalised histogram, in the following called a frequency curve, with an area under the curve (AUC) of 100\% (see Figure 14a). The frequency curves of healthy subjects show distinct high peaks while the frequency curves of patients with severe COPD are low and wide. The normalisa- 
tion was performed in order to compare frequency curves based on different lung sizes (different numbers of voxels) visually. The relative numbers of $\mathrm{CV}$ values in bins of width of $1 \%$ were used to construct the frequency curves. The area under the frequency curve (AUC) for $\mathrm{CV}$ values greater than a threshold value for $\mathrm{CV}$, called $\mathrm{CV}_{\mathrm{T}}$, was defined as $\mathrm{AUC}\left(\mathrm{CV}_{\mathrm{T}}\right)$ and expressed as the percentage of the total AUC (Figure 15). The final $\mathrm{AUC}\left(\mathrm{CV}_{\mathrm{T}}\right)$ value is used for comparison of the amount of heterogeneity in-between groups or individual subjects.

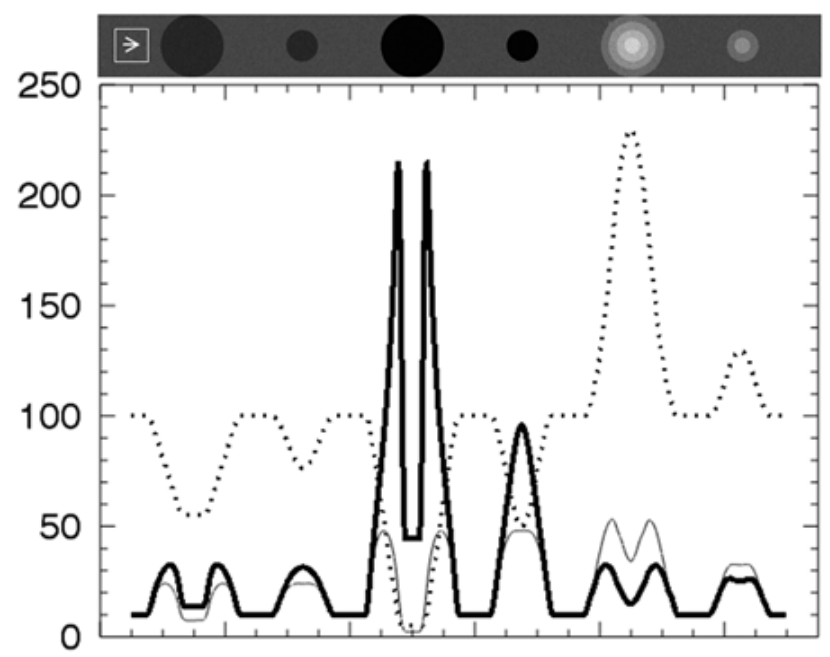

Figure 13. An illustration of the behaviour of the coefficient of variance, CV, (\%), standard deviation and mean value. At the top is a slice through a three dimensional geometry with a background mean activity level of 100 and spheres from the left to the right at 55, 55, 5, 5, 300 (in the centre) and 200 (in the centre) with Poisson noise incorporated. Outlined at the top is also the cubic kernel used to step through the geometry calculating the mean (dotted line), standard deviation (solid thin line) and CV (solid thick line) for the enclosed voxels. The calculated values were plotted opposite the central position of the kernel.

\section{Analysis parameters}

Important factors for analysis using the $\mathrm{CV}_{\mathrm{T}}$-method are the choice of inclusion or exclusion of the periphery layer, the size of the kernel and the threshold value. Together with the acquisition and reconstruction parameters the analysis parameters determine the ability of the acquisition method to discriminate between various ventilation activity distributions. 

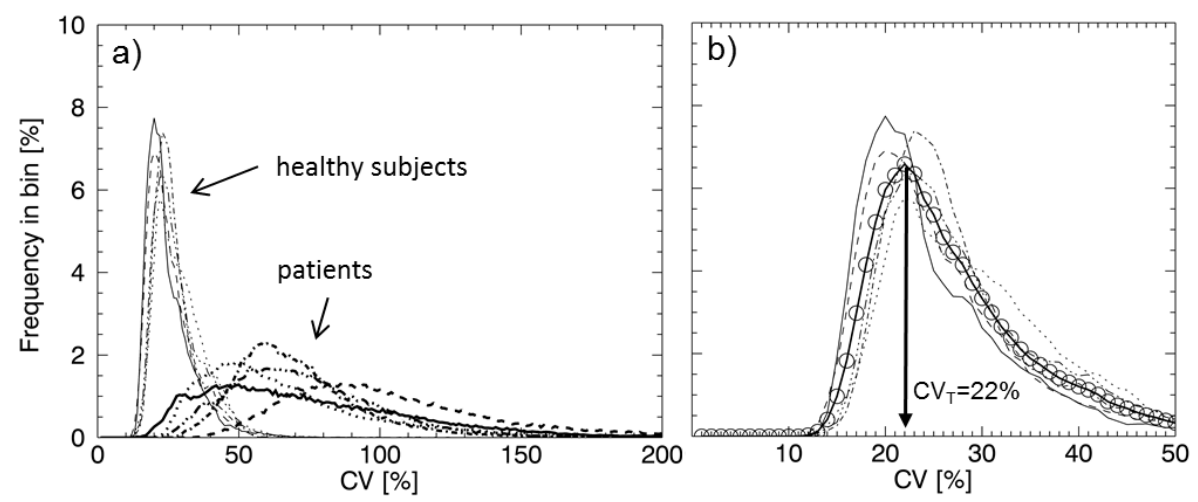

Figure 14. Frequency curves of the CV values for the human study in paper II. a) The frequency curves of the $C V$ values corresponding to healthy subjects (thin lines), and patients with bold line styles. b) An expanded view of the frequency curves for the healthy subjects and their mean frequency curves (bold line with open circles). The arrow shows the CV threshold value for the human study at 22\%. (Paper II and poster ii)

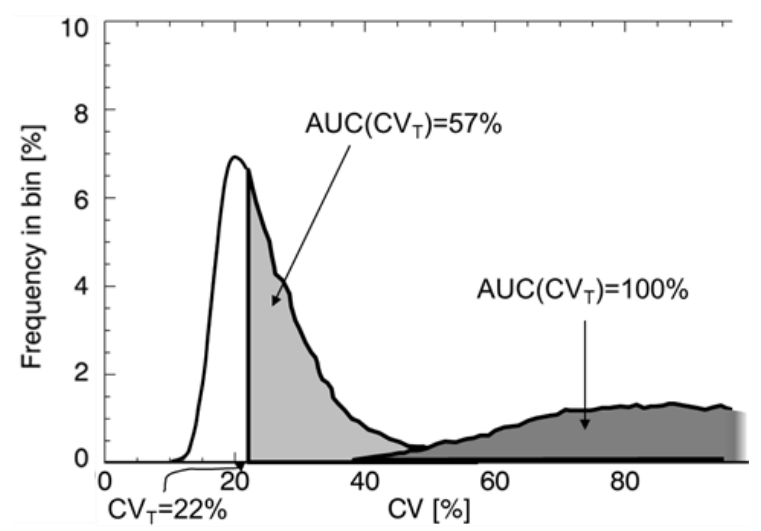

Figure 15. The area under the curve (AUC) for $C V$-values greater than $C V_{T}=22 \%$ for subjects in paper II; a healthy subject (light grey), a patient (dark grey). (Poster ii) 


\section{Evaluation of the $\mathrm{CV}_{\mathrm{T}}$-method using phantoms}

The ability of the $\mathrm{CV}_{\mathrm{T}}$ method to discriminate between a simulated "healthy" lung and a range of simulated COPD lungs with varying severity was evaluated (paper II).

\section{Material and Methods}

The NCAT software was used to generate a phantom with a lung volume corresponding to a 65 -year-old male $(70 \mathrm{~kg}, 180 \mathrm{~cm})$ in a supine position and in the middle of the respiratory cycle (Ibanez and Raurich, 1982; Quanjer et al., 1993). The respiratory motion and heartbeat of the phantom were employed throughout the study, i.e. the phantom was dynamic.

Created activity distributions aimed to mimic mild to moderate changes of COPD, and therefore small lesions were of interest. The lesions modelled had a diameter of 1 and $2 \mathrm{~cm}$. Lacking previous studies on the distribution of ventilation inhomogeneities in mild COPD, we assumed that COPD lesions are either evenly distributed in the whole lung volume or centred in clusters. The latter case is therefore partly uniform/ partly heterogeneous. Coronal slices of activity distributions used in paper II are shown in Table 1. The activity distributions listed are the simulated "healthy" lung with uniform activity distribution throughout the lung and eight simulated COPD lung changes of varying severity of disease. For distribution 2 the reduction of ventilation of $50 \%$ in $10 \%$ of the lung volume corresponds to a $5 \%$ total reduction of the functioning lung tissue. Since the normal range of spirometric variables is about $15-20 \%$, a distribution such as distribution 2 is assumed to be representative for a case of mild COPD. The distribution observed by the gamma camera includes motion artefacts particularly in the lower parts of the lung as illustrated in the slices.

The projections from the activity distributions were generated using the SIMIND software. The projection data incorporated the effects of non-uniform attenuation and scatter. The simulations were made for a GE Infinia gamma camera (9.5-mm thick NaI detector) equipped with a LEHR collimator.

The mean number of counts in the simulated projections was set to $3.635 \times$ $10^{6}$, for each activity distribution. This mean number of counts corresponds to a virtual administered activity of $125 \mathrm{MBq}$ (128 projections á $10 \mathrm{~s}, 22.7 \mathrm{cps} / \mathrm{MBq})$. For each activity distribution, 20 noise realisations were created.

Reconstructions were performed using OSEM including attenuation, scatter and CDR compensation. Ten iterations and 16 subsets were used, i.e. 160 updates. The side length of the cubic voxels in the reconstructed image was 3.30 $\mathrm{mm}$. The reconstructed images were post-filtered with a Butterworth filter with a cut-off frequency of $0.5 \mathrm{~cm}^{-1}$ and a power of 6 . 
Table 1. Phantom activity distribution notations and their descriptions.

\begin{tabular}{|c|c|c|c|c|}
\hline & $\begin{array}{c}\text { Distribution } \\
\text { notation }\end{array}$ & Coronal slice & Distribution description & $\begin{array}{c}\text { Total reduction } \\
\text { of ventilation } \\
{[\%]}\end{array}$ \\
\hline $\begin{array}{l}\text { Simulated } \\
\text { normal }\end{array}$ & $\begin{array}{l}\text { Uniform } \\
\text { "healthy" }\end{array}$ & & $\begin{array}{l}\text { 1: Homogeneous activity distribu- } \\
\text { tion }\end{array}$ & 0 \\
\hline \multirow[t]{8}{*}{$\begin{array}{l}\text { Simulated } \\
\text { COPD }\end{array}$} & $\begin{array}{l}1 \mathrm{~cm}^{50 \%} 10 \% \\
\text { evenly } \\
\text { "Mild COPD" }\end{array}$ & & $\begin{array}{l}\text { 2: Lesions with a diameter of } 1 \mathrm{~cm} \\
\text { with } 50 \% \text { activity concentration, } \\
\text { evenly distributed over the lung } \\
\text { volume, occupying } 10 \% \text { of the total } \\
\text { lung volume. }\end{array}$ & 5 \\
\hline & $\begin{array}{l}1 \mathrm{~cm}^{50 \%} 12 \% \\
\text { centred }\end{array}$ & & $\begin{array}{l}\text { 3: Lesions with a diameter of } 1 \mathrm{~cm} \\
\text { with } 50 \% \text { activity concentration, } \\
\text { centred to the large bronchial tubes, } \\
\text { occupying } 12 \% \text { of the total lung } \\
\text { volume. }\end{array}$ & 6 \\
\hline & $\begin{array}{l}1 \mathrm{~cm}^{0 \%} 10 \% \\
\text { evenly }\end{array}$ & & $\begin{array}{l}\text { 4: Lesions with a diameter of } 1 \mathrm{~cm} \\
\text { with } 0 \% \text { activity concentration, } \\
\text { evenly distributed over the lung } \\
\text { volume, occupying } 10 \% \text { of the total } \\
\text { lung volume. }\end{array}$ & 10 \\
\hline & $\begin{array}{l}1 \mathrm{~cm}^{0 \%} 12 \% \\
\text { centred }\end{array}$ & & $\begin{array}{l}\text { 5: Lesions with a diameter of } 1 \mathrm{~cm} \\
\text { with } 0 \% \text { activity concentration, } \\
\text { centred to the large bronchial tubes, } \\
\text { occupying } 12 \% \text { of the total lung } \\
\text { volume. }\end{array}$ & 12 \\
\hline & $\begin{array}{l}2 \mathrm{~cm}^{50 \%} 10 \% \\
\text { evenly }\end{array}$ & & $\begin{array}{l}\text { 6: Lesions with a diameter of } 2 \mathrm{~cm} \\
\text { with } 50 \% \text { activity concentration, } \\
\text { evenly distributed over the lung } \\
\text { volume, occupying } 10 \% \text { of the total } \\
\text { lung volume. }\end{array}$ & 5 \\
\hline & $\begin{array}{l}2 \mathrm{~cm}^{0 \%} 10 \% \\
\text { evenly }\end{array}$ & & $\begin{array}{l}\text { 7: Lesions with a diameter of } 2 \mathrm{~cm} \\
\text { with } 0 \% \text { activity concentration, } \\
\text { evenly distributed over the lung } \\
\text { volume, occupying } 10 \% \text { of the total } \\
\text { lung volume. }\end{array}$ & 10 \\
\hline & $\begin{array}{l}2 \mathrm{~cm}^{50 \%} 48 \% \\
\text { evenly }\end{array}$ & & $\begin{array}{l}\text { 8: Lesions with a diameter of } 2 \mathrm{~cm} \\
\text { with } 50 \% \text { activity concentration, } \\
\text { evenly distributed over the lung } \\
\text { volume, occupying } 48 \% \text { of the total } \\
\text { lung volume. }\end{array}$ & 24 \\
\hline & $\begin{array}{l}2 \mathrm{~cm}^{25 \%} 48 \% \\
\text { evenly }\end{array}$ & & $\begin{array}{l}\text { 9: Lesions with a diameter of } 2 \mathrm{~cm} \\
\text { with } 25 \% \text { activity concentration, } \\
\text { evenly distributed over the lung } \\
\text { volume, occupying } 48 \% \text { of the total } \\
\text { lung volume. }\end{array}$ & 36 \\
\hline
\end{tabular}

Coronal slices of the phantom including simulated motion artefacts. 
The lung voxels of the phantom in the middle of the respiratory cycle were set to be the segmented lung. A one-voxel-wide layer of the lung was eroded from the periphery of the segmented lung, creating a reduced lung volume with most of the periphery effect excluded. For the CV calculations a $3 \times 3 \times 3 \mathrm{~cm}^{3}$ (i.e. $9 \times 9 \times 9$ voxels) cubic kernel stepped through the reconstructed lung volume. The threshold value $\left(\mathrm{CV}_{\mathrm{T}}\right)$ was the mode value of the mean frequency curve based on the 20 noise realisations of the "healthy" uniform activity distribution (distribution 1 in Table 1). $\mathrm{AUC}\left(\mathrm{CV}_{\mathrm{T}}\right)$ was then calculated for all activity distributions. The group of $20 \mathrm{AUC}\left(\mathrm{CV}_{\mathrm{T}}\right)$ values, based on the 20 noise realisations of the simulated "healthy" distribution, was compared with the corresponding groups of each simulated COPD distributions. For the comparison, the nonparametric Mann-Whitney U test was used.

\section{Results and discussion}

The $\mathrm{CV}_{\mathrm{T}}$ method was able to assess even minor COPD changes by using the $\operatorname{AUC}\left(\mathrm{CV}_{\mathrm{T}}\right)$ value, as a global value of ventilation inhomogeneities, and to discriminate these changes from a model of a "healthy" homogeneous lung.

A significant difference $(\mathrm{p}<0.001)$ was found between $\mathrm{AUC}\left(\mathrm{CV}_{\mathrm{T}}\right)$ values for the "healthy" and "mild COPD" lungs (distributions 1 and 2, in Table 1) (Figure 16). That is, the method identified a total reduction of ventilation of approximately $5 \%$. Distribution 2 consists of $1 \mathrm{~cm}$ spheres. A much larger difference was found between distribution 1 and 6 . This is because distribution 6 consists of 2 $\mathrm{cm}$ spheres, which are easier for the SPECT system to resolve. The AUC $\left(\mathrm{CV}_{\mathrm{T}}\right)$ values are based on the frequency curves shown in Figure $17 . \mathrm{CV}_{\mathrm{T}}=20.5 \%$. The $5 \%$ reduction of ventilation is not visible to the human eye in the reconstructed image, as shown in Figure 18 (row 2, column B).
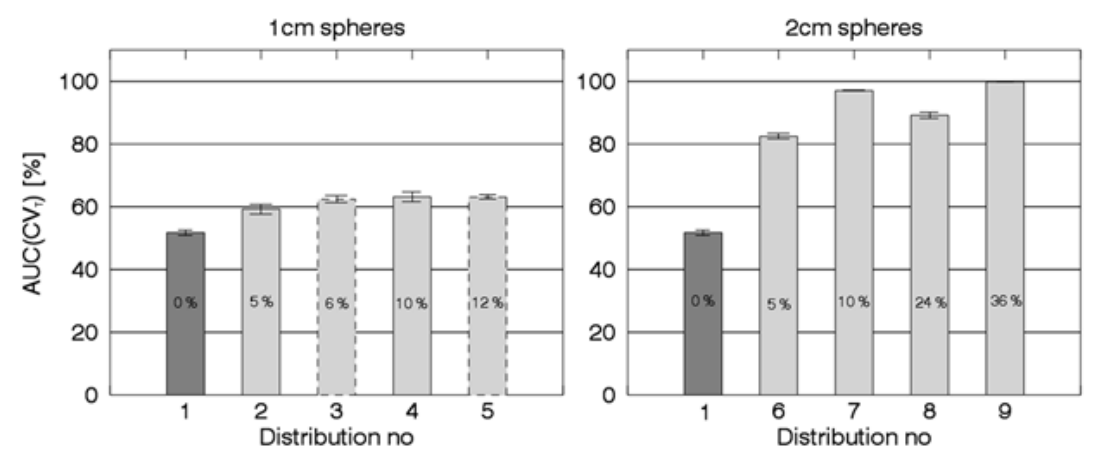

Figure 16. The mean $A U C\left(C V_{T}\right)$ value for each activity distribution as listed in Table 1. The dark grey bars show the simulated "healthy" distribution, and the light grey bars show simulated COPD distributions. Bars with dashed outline correspond to clustered distributions (partly uniform/partly heterogeneous). The total reduction of ventilation for each distribution is written in the bars. The error bars show a 95\% confidence interval (CI), based on the 20 noise realisations. 


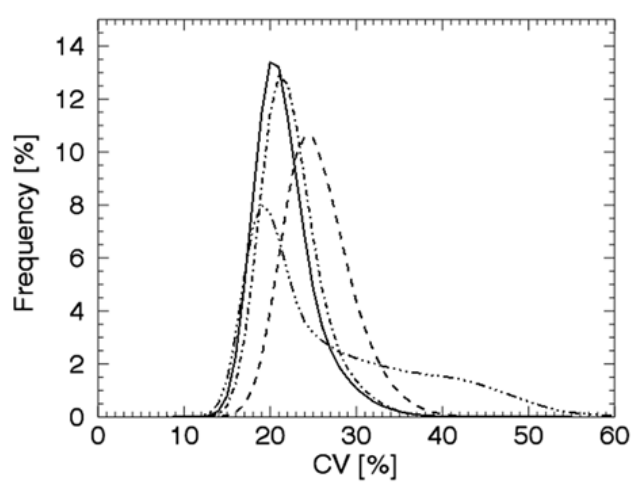

Figure 17. Mean frequency curves of the $C V$ values for the simulated distributions. The mean frequency curves of 20 noise realisations of the $C V$ values corresponding to distribution 1, the simulated "healthy" distribution (solid line); distribution 4, '1 cm $10 \%$ evenly' (dash-dotted); distribution 5 , ' $1 \mathrm{~cm}^{0 \%} 12 \%$ centred' (dash-dot-dot-dotted); and distribution 8, ' $2 \mathrm{~cm}^{50 \%} 48 \%$ evenly' (dashed). Only four cases out of nine (see Table 1) are shown for clarity.

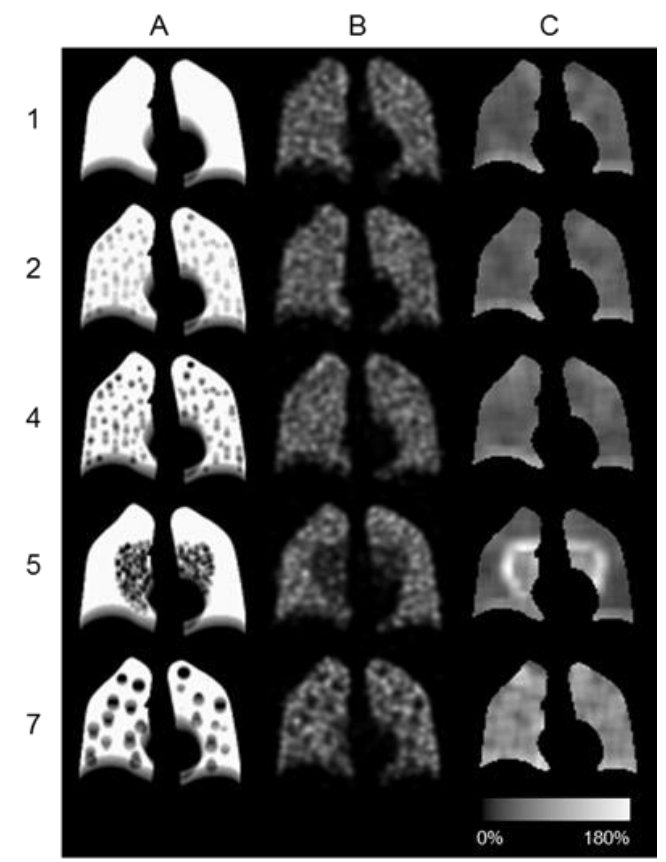

Figure 18. The figure shows reconstructed images and CV matrices for simulated activity distributions. Each row shows coronal slices based on the activity distributions 1, 2, 4, 5 and 7 in Table 1, A); the activity distribution, B); a filtered reconstruction and, C); corresponding parametric image. 
It is important to point out that the result of this study is based on equal amount of total activity in each distribution. Therefore, the "healthy" lung will have a lower count level compared to the "healthy" surroundings of the heterogeneous activity distributions. If instead the "healthy" regions of the COPD distributions had been given the same activity concentration as the "healthy" distribution, the COPD frequency curves would be shifted somewhat to the right on the CV axis in Figure 17. This would result in an even greater difference between the uniform "healthy" distribution and the simulated COPD distributions in Figure 16. The idea of equalising "healthy" volumes between subjects was first thought of later on when creating the compensation method.

\section{Optimisation of the included parameters when using the $\mathrm{CV}_{\mathrm{T}}$-method}

In paper III the acquisition, reconstruction and analysis parameters were optimised to provide the best chance for the $\mathrm{CV}_{\mathrm{T}}$-method to successfully differentiate between two activity distributions. The ultimate test of the applicability of the $\mathrm{CV}_{\mathrm{T}}$-method will require access to a large group of well-defined healthy subjects and a large group of well-defined patients with mild COPD. Lacking access to these groups of subjects, we used Monte Carlo simulated lung-SPECT images of phantom lungs instead. The parameters studied were activity level, collimator, number of OSEM updates, cut-off frequency of the noise reduction filter, volume of analysis and size of the cubic kernel.

\section{Material and Methods}

In paper II we evaluated the outcome of the $\mathrm{CV}_{\mathrm{T}}$-method using a variety of different simulated COPD activity distributions. The COPD distribution that was most difficult to separate from the simulated "healthy" distribution was chosen in this study, i.e. the "mild COPD" (distribution 2 in Table 1).

Simulations were made for a GE Infinia gamma camera $(0.95 \mathrm{~cm}$ thick NaI detector) equipped with a LEHR and a LEGP collimator. The mean number of counts in the simulated projections for both activity distributions ("healthy" and "mild COPD") was set according to Table 2. These values correspond to administered activities of 25 and $125 \mathrm{MBq}$ using LEGP and LEHR collimators, respectively (paper II). For each activity distribution, 40 noise realisations were created.

OSEM reconstructions were performed using 16 subsets and 10 different numbers of iterations (see Table 3 ) incorporating attenuation, scatter and CDR compensation. The side length of a voxel in a reconstructed image was $0.33 \mathrm{~cm}$. The reconstructed images were post-filtered with a Butterworth filter with four different cut-off frequencies and a power of 6, or not filtered at all (see Table 3). 
Table 2. The mean number of counts in the 128 simulated projections for the two collimators and two levels of administered activity of ${ }^{99 m} \mathrm{Tc}$.

Administered activity

\begin{tabular}{ccc} 
& $25 \mathrm{MBq}$ & $125 \mathrm{MBq}$ \\
\hline LEGP & $1.23 \times 10^{6}$ & $6.14 \times 10^{6}$ \\
LEHR & $0.73 \times 10^{6}$ & $3.64 \times 10^{6}$
\end{tabular}

Table 3. Variables used in the study.

Acquisition

Activity levels

25 and $125 \mathrm{MBq}$

Collimators

LEHR and LEGP

Reconstruction

Number of iterations

$2,4,6, \ldots, 20$ with 16 subsets, i.e. $32-320$ updates

Cut-off frequencies

$0.4,0.5,0.6,0.7 \mathrm{~cm}^{-1}$ and no filtering

Analysis

Side lengths of the kernel $1.0,1.7,2.3$ and $3.0 \mathrm{~cm}$ (i.e. $3,5,7$ and 9 voxels)

Volume of analysis The whole lung and the reduced lung

The lung voxels of the phantom in the middle of the respiratory cycle were set to be the segmented lung. A one-voxel-wide layer of the lung was eroded from the periphery of the segmented lung, creating a reduced lung volume with most of the periphery effect excluded. In the evaluation, four side lengths of the kernel were used (see Table 3). Each combination of activity level, collimator, number of updates, cut-off frequency of the noise reduction filter and side length of the kernel is here called a design. For each design and activity distribution, 40 frequency curves of $\mathrm{CV}$ values based on the 40 noise realisations were generated. For each design, the frequency curves of the "healthy" distribution generated a mean frequency function. Its mode value was then used as the threshold, $\mathrm{CV}_{\mathrm{T}}$, value. 
For each design and activity distribution, $40 \mathrm{AUC}\left(\mathrm{CV}_{\mathrm{T}}\right)$-values were obtained from the 40 frequency functions. For the comparison of the two activity distributions, the non-parametric Mann-Whitney U-test was used. The designs were placed in order of rank based on resulting p-values. P-values corresponding to negative Z-statistic values were not included in the ranking. The lowest $\mathrm{p}$ value corresponds to the greatest separation between the $\operatorname{AUC}\left(\mathrm{CV}_{\mathrm{T}}\right)$-values for the "healthy" and "mild COPD" distributions.

\section{Results and discussion}

The study gave two results, one for each volume of analysis (column left and right in Figure 19). The greatest separation was achieved by using a total of at least $3.64 \times 10^{6}$ counts in the projections (named $125 \mathrm{MBq}$ ), using an LEHR collimator together with a Butterworth power 6 noise-reduction filter with a cut-off frequency of $0.6-0.7 \mathrm{~cm}^{-1}$. When the whole lung was analysed, 64 reconstruction updates and a kernel with a side length of $1.0 \mathrm{~cm}$ gave the best result. For the reduced lung volume a greater number of updates and a larger kernel size are needed. The reduced lung resulted in lower p-values for LEHR-125 MBq-designs compared to when the whole lung was analysed.

The ten designs generating the lowest $\mathrm{p}$-values for the whole lung are listed in Table 4. The lowest $\mathrm{p}$-value for a LEGP-125MBq-design for the whole lung was $\mathrm{p}=8.0 \times 10^{-4}$, and for the reduced lung $\mathrm{p}=2.0 \times 10^{-8}$. The other groups of designs, LEGP-25MBq-designs and LEHR-25MBq-designs, had higher p-values.

For quantitative assessment of small and less distinct lesions, as in this study, a high-count density and a high spatial resolution were needed. The LEGP collimator could not resolve the small lesions sufficiently for either of the two activity levels. The LEHR collimator together with $25 \mathrm{MBq}$ resulted in an excessively low count level. Increasing the number of counts increased the separation between the imaged "healthy" and "mild COPD" distributions. Therefore, in a clinical setting, it is important to reach the higher level of inhaled activity. To increase the separation using the LEHR collimator further than shown in this study, even higher count levels than $3.64 \times 10^{6}$ are needed.

Generally, 64 and 96 updates were found optimal for LEHR-125MBq designs, which does not contradict the result from paper II. An even higher number of updates would increase the contrast recovery, but at the same time increase the noise level to an extent that diminishes the advantage of increased contrast. A soft noise reduction filter with a cut-off frequency of $0.6-0.7 \mathrm{~cm}^{-1}$ was found optimal. 
a)

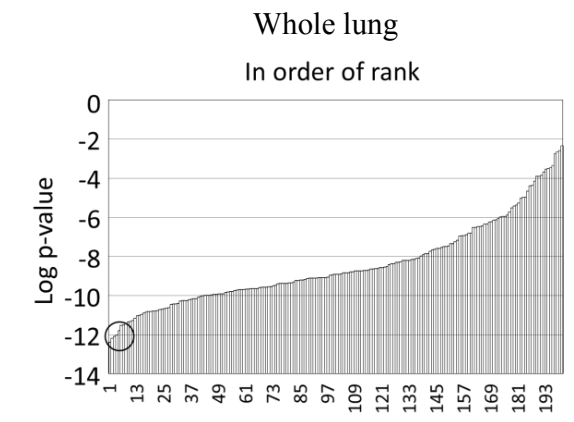

b)

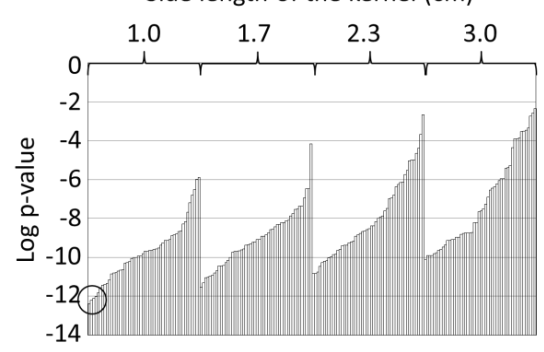

c)

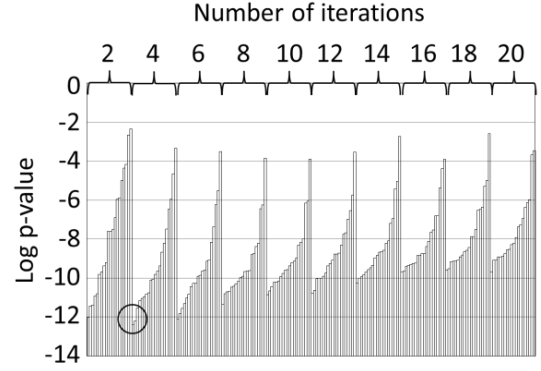

d)

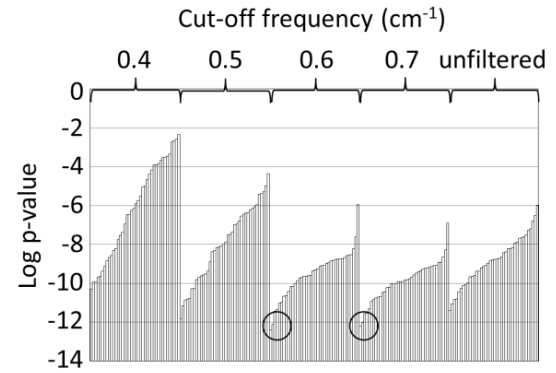

Reduced lung

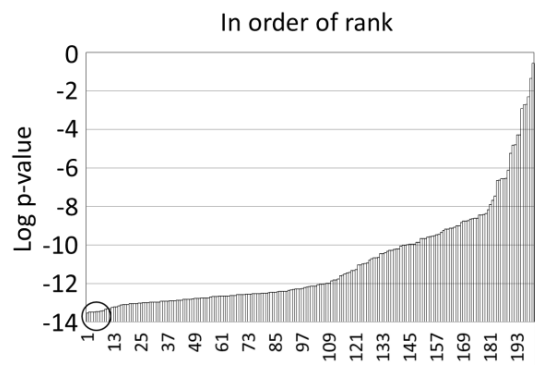

Side length of the kernel $(\mathrm{cm})$

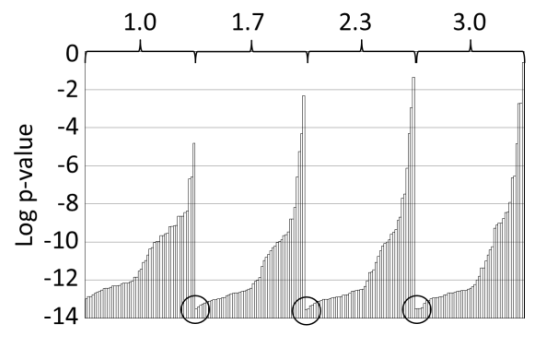

Number of iterations

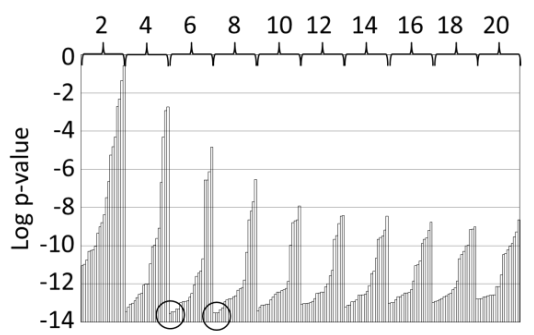

Cut-off frequency $\left(\mathrm{cm}^{-1}\right)$

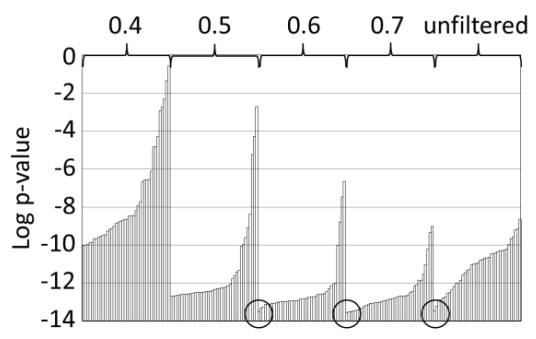

Figure 19. The LEHR-125MBq-designs for the whole lung is shown in the left column and for the reduced lung in the right column. In row a) the designs are placed in order of rank based on resulting p-values. In row b) the designs are grouped by kernel size, row c) by iteration number (16 subsets) and in row d) by cut-off frequency of the Butterworth filter. The lowest $p$-values are encircled. 
Table 4. The 10 designs generating the lowest $p$-values, for the whole lung, ranked in order of increasing $\mathrm{p}$ value. All designs use LEHR collimator and 125 MBq.

\begin{tabular}{ccccc}
\hline $\begin{array}{c}\text { Order of } \\
\text { rank }\end{array}$ & $\begin{array}{c}\text { Side length } \\
\text { of the kernel } \\
(\mathrm{cm})\end{array}$ & $\begin{array}{c}\text { No of } \\
\text { iterations } \\
\text { (updates) }\end{array}$ & $\begin{array}{c}\text { Cut-off } \\
\text { frequency } \\
\left(\mathrm{cm}^{-1}\right)\end{array}$ & p-value \\
\hline 1 & 1.0 & $4(64)$ & 0.6 & $4.1 \times 10^{-13}$ \\
2 & 1.0 & $4(64)$ & 0.7 & $6.2 \times 10^{-13}$ \\
3 & 1.0 & $6(96)$ & 0.6 & $7.9 \times 10^{-13}$ \\
4 & 1.0 & $2(32)$ & 0.7 & $9.4 \times 10^{-13}$ \\
5 & 1.0 & $6(96)$ & 0.5 & $1.5 \times 10^{-12}$ \\
6 & 1.0 & $6(96)$ & 0.7 & $2.8 \times 10^{-12}$ \\
7 & 1.7 & $4(64)$ & 0.7 & $3.0 \times 10^{-12}$ \\
8 & 1.0 & $2(32)$ & 0.6 & $3.7 \times 10^{-12}$ \\
9 & 1.0 & $2(32)$ & unfiltered & $4.2 \times 10^{-12}$ \\
10 & 1.0 & $8(128)$ & 0.6 & $4.5 \times 10^{-12}$ \\
\hline
\end{tabular}

Using the optimised parameter values obtained in this work improved the ability of the $\mathrm{CV}_{\mathrm{T}}$-method compared to previously published results on simulated distributions (paper II). The optimisation reduced the resulting $\mathrm{p}$-value from $5.8 \times 10^{-13}$ to $2.8 \times 10^{-14}$ for the "mild COPD" distribution and the reduced lung volume.

It could be that clustered lesions, as in distribution 3 in Table 1, is more likely to be found in individuals with mild ventilation heterogeneities than uniformly distributed lesions. Then again, according to the evaluation of the $\mathrm{CV}_{\mathrm{T}}$-method using phantoms, the clustered distribution with similar total reduction of ventilation as the "mild COPD" distribution was somewhat easier for the method to resolve. Thus, the optimisation was performed on the most difficult case.

For each comparison of the "healthy" and "mild COPD" activity distributions, two $\mathrm{AUC}\left(\mathrm{CV}_{\mathrm{T}}\right)$ means with $95 \% \mathrm{CI}$ error bars can be generated based on their 40 noise realisations. Even though the p-values presented in this optimisation were low, the increased separation between $\operatorname{AUC}\left(\mathrm{CV}_{\mathrm{T}}\right)$ means down to pvalues of at least $1 \times 10^{-13}$ were visible.

Applying the optimised paramters found in this stydy to the human study in paper II did not improve (or impair) the resulting p-value. This was, due to the low number of subjects and the Mann-Whitney U-test. No improvements can be identified using p-values since the lowest value achievable with the MannWhitney U-test was already reached before the optimisation in this work. Visual inspection of bar plots shows equal results. 


\section{The threshold value, $\mathrm{CV}_{\mathrm{T}}$}

In paper II (evaluation on phantoms and COPD patients) and III (optimisation) the threshold value, $\mathrm{CV}_{\mathrm{T}}$, was defined as the most frequently occurring $\mathrm{CV}$ value (the mode or modal value) of the frequency curve of the healthy normal. For the human study in paper II this meant that the threshold value was defined as the mode value of the mean frequency curve of the healthy subjects, as shown in Figure 14b. In the simulated COPD studies (paper II and III), the healthy normal was the mean frequency curve of a number of noise realisations of the uniform activity distribution.

The strategy of using the mode as the threshold value was the result of an optimisation of finding the largest separation between the $\mathrm{AUC}\left(\mathrm{CV}_{\mathrm{T}}\right)$ values of the uniform ("healthy") distribution (distribution 1 in Table 1, page 39) and a heterogeneous distribution with ventilation defects ("early COPD") (distribution 2 in Table 1) in a phantom lung. The optimal $\mathrm{CV}_{\mathrm{T}}$ value was found to be the mode plus about $1 \%$, but the mode value was used for simplicity. The frequency curve of this "early COPD" distribution was closest to the frequency curve of the "healthy" distribution of all our evaluated phantom distributions. With the objective to distinguish between healthy and mild lung function reduction it was logical to base the optimisation of the threshold value on this "early COPD" distribution. This "early COPD" distribution consisted of a large number of small uniformly distributed spherical ventilation defects with reduced activity compared to the uniform surrounding activity level. Due to the limited spatial resolution of the SPECT system and movement of the spheres due to respiratory motion and heartbeat the edges of the spheres were blurred in the reconstructed image. Therefore, the lowest CV values found in the "healthy" uniform distribution were not found in the "early COPD" distribution. The resulting frequency curve of the "early COPD" distribution had approximately the same shape as the one of the "healthy" distribution but shifted towards higher CV-values. When applied on phantom distributions, and focusing on two curves with the same shape separated by a small shift, the mode value of the "healthy" frequency curve was a good threshold value.

Experienced by the resulting frequency curves of human subjects in paper II (Figure 14a) and paper IV (Figure 20) we realised that a threshold value based on the intersection between frequency curves of the patients and the healthy subjects would increase the separation between the $A U C\left(C_{T}\right)$ values of healthy volunteers and patients. Therefore, in paper IV we present the results of using both the mode value and the intersection-CV value as the threshold value. 


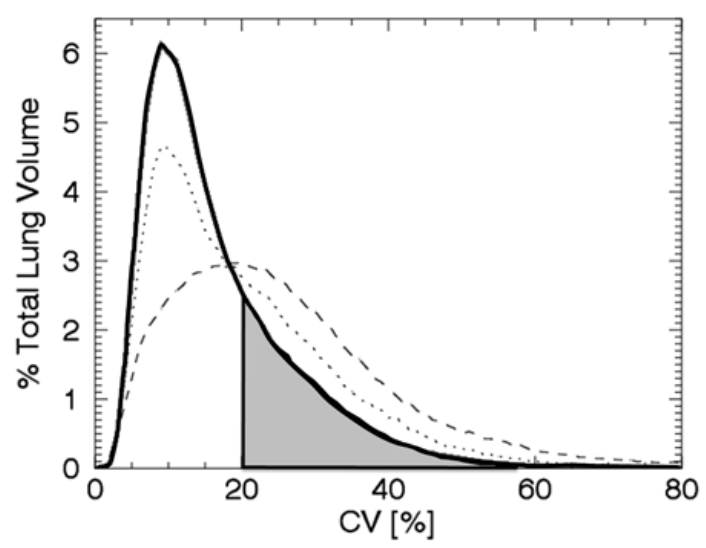

Figure 20. Frequency curves of patient $P 1$ (dotted line) and $P 2$ (dashed line) together with the mean curve (solid line) from paper IV. AUC for $C V$-values greater than $20 \%$, i.e. $A U C(C V>20 \%)$, for the mean curve is marked as light grey. (Paper IV)

\section{Volume of interest and kernel size}

High CV values will always be found in the periphery of the healthy lung due to the periphery effect. Lung peripheries with low activity lesions will instead give lower $\mathrm{CV}$ values. High $\mathrm{CV}$ values due to healthy peripheries reduce the differences between frequency curves from healthy and unhealthy activity distributions; i.e. they reduce the separation between corresponding $\mathrm{AUC}\left(\mathrm{CV}_{\mathrm{T}}\right)$ values. Therefore, in paper II the CV analysis was performed in a volume that had part of the peripheries effect excluded. One peripheral voxel layer was eroded from the total lung volume. Exclusion of a one-voxel layer, however, removes as much as $21 \%$ of the phantom lung and about $25 \%$ of the human lung parenchyma from the analysis, which is a disadvantage since small lesions in the periphery might not be detected. In paper IV we reconsidered the choice of lung periphery exclusion. We did not want to ignore the potential information from the periphery of the lung and therefore used the whole lung in the analysis.

The kernel size used in paper II had a side length of $3 \mathrm{~cm}$, including 729 voxels. Such a large kernel was found appropriate together with the reduced lung volume. None of the acquisition, reconstruction and analysis parameter values used in paper II were formally optimized but were good enough for the purpose of demonstrating the usefulness of the suggested method. Later, the optimisation performed in paper III showed that a large kernel size should be used in combination with eroded periphery. The optimisation work also showed that a small kernel size, with a side length of $1.0 \mathrm{~cm}$, should be used when the periphery was included in the analysis. This small kernel was therefore used in the pilot study in paper IV. 


\section{The compensation method}

With experience from the studies with healthy human subjects in paper II and IV we noticed that the varying amount of inhaled Technegas and varying sizes of lungs resulted in shifts of the frequency curves on the CV-axis between subjects. This was due to varying number of deposited ${ }^{99 \mathrm{~m}} \mathrm{Tc}$ particles per unit volume between subjects (Figure 21a). Using Monte Carlo simulations and various predefined activity distributions in a phantom lung, properties of frequency curves was analysed. We evaluated the frequency curves, which were based on an entirely uniform (homogeneous) activity distribution, and a partly uniform / partly heterogeneous activity distribution (distributions 1 and 5 in Table 1, page 39). We noticed that lung regions with uniform and equal activity concentration in both distributions generated curves that started at the same position to the left of the $\mathrm{CV}$-axis and had the same mode value (Figure $21 \mathrm{~b}$ ). The low CV values in the peak of the frequency curve of a healthy subject correspond to the most uniform part of the activity distribution, that ought to be the most healthy and wellventilated part of the lung.

To make it possible to discriminate between subjects with less pronounced differences in ventilation heterogeneity using the $\mathrm{CV}_{\mathrm{T}}$-method a compensation has to be performed. Such compensation should reduce the subject-to-subject variation, making the $\mathrm{CV}$ values from healthy lung volumes of different subjects comparable (paper IV).

One strategy to get frequency curves of human subjects with the same mode value could be to beforehand calculate the amount of Technegas to be inhaled, if we knew the volume the Technegas was going to be distributed in. Therefore, various volumes were evaluated, such as $\mathrm{VC}, \mathrm{VC} / \mathrm{TLC}$ and the lung volume according to the CT scan, retrospectively. The volumes were evaluated together with an estimation of deposited Technegas activity based on the perfusion acquisition performed with a known amount of activity. This was done without finding a good correlation to measured mode values for any of the evaluated volumes. Furthermore, the procedure we used to estimate the inhaled activity at the time of examination is imprecise. Supposing the amount of activity to be inhaled could be calculated beforehand, we would need a more precise procedure to estimate the inhaled activity at the time of examination to be able to use that information.

$\mathrm{AUC}\left(\mathrm{CV}_{\mathrm{T}}\right)$ values based on the original frequency curves in Figure $21 \mathrm{a}$ of the healthy subjects could be used to demonstrate a significant differense from patients with severe COPD. While, if smaller differences are to be found the frequency curves need to be based on more equal conditions. Therefore, a compensation method was developed and introduced in paper IV in order to compensate for between subject-to-subject variations of activity uptake. The compensation method was described in the Appendix to paper IV. 

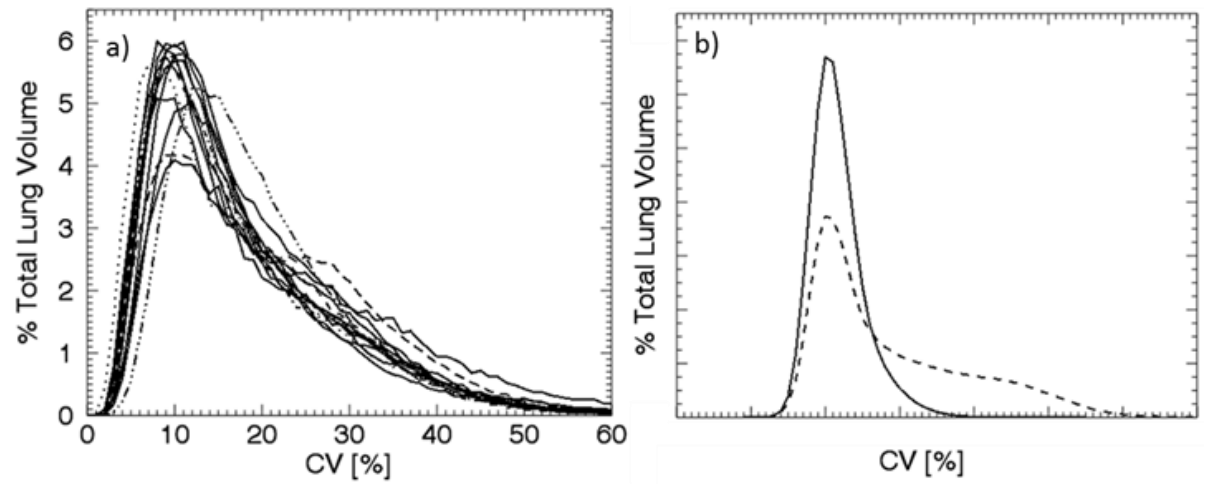

Figure 21. Shown in a) are the between subjects varying starting points of the original frequency curves at low $C V$-values and their between subjects varying mode values of the 14 examined healthy subjects in paper IV. Notice the same starting point and mode value in b) for the mean frequency curves based on an entirely uniform activity distribution in the phantom lung (solid black line, distribution 1 in Table 1) and partly uniform/partly heterogeneous distribution (dotted black line, distribution 5 in Table 1). Both have equal activity concentration in their uniform regions and the mean curves are based on 20 noise realisations (from Appendix paper IV).

The compensation was performed in two steps. First, a shift of all frequency curves was made so that the left flank of the frequency curves passed through a well-chosen point in the plot, namely a frequency of $0.5 \%$ (i.e. $0.5 \%$ of the total lung volume) at $3 \mathrm{CV} \%$ (hereafter called the fix point). This point was chosen as many of the uncompensated frequency curves passed close to this point (Figure 21a). Performing this shift, we assume that the length of each shift is due only to statistical noise variations between subjects and that each subject has a healthy lung region large enough to generate a distinct peak in the frequency curve. For a subject with severe COPD this assumption might not be met and therefore the applied shift will remove true measured heterogeneities from the analysis. This will also be the case for a supposed much less diseased subject with an activity distribution like the simulated "early COPD" (distribution 2 in Table 1), which generates a distinct peak based on subtle but true heterogeneities. Since the aim of this thesis is to find mild ventilation heterogeneities in subjects with normal lung function, according to lung function tests, we assume that subjects with no healthy lung regions are rare. Subjects with an activity distribution like the simulated "early COPD" will not be identified in an analysis that uses this compensation method.

Studying the joint points of the frequency curves in Figure $21 \mathrm{~b}$ one can question why the fix point was not chosen further down the left flank of the peaks. This can be explained by the fact that the frequency curves in Figure $21 \mathrm{~b}$ are mean curves of twenty noise realisations while the subjects' curves in Figure $21 \mathrm{a}$ are just one noise realisation. Futhermore, low frequency values are based on 
few CV-measurements. This means that the subjects' low frequency values, down the left flank of the peak, are less stable compared to the simulated once in Figure 21b. Therefore, a more solid fix point was chosen (i.e. based on a larger number of $\mathrm{CV}$-measurements).

If the compensation incorporated a shift only, the resulting frequency curves would have a more correct starting point compared to prior the compensation but with a less correct shape. This is illustrated in Figure 22. Frequency curves based on a uniform distribution (distribution 1 in Table 1) but with two different activity levels demonstrate different shapes and positions on the $\mathrm{CV}$-axis. No periphery effect is included in the curves. This change in shape would also be the case in more complex distributions such as in the lung of a human subject. If the healthy region of a lung received an extremely low amount of deposited ${ }^{99 \mathrm{~m}} \mathrm{Tc}$ particles per unit volume the rest of the lung also did. Therefore, if one individual made two ventilation SPECT examinations, inhaling different amounts of Technegas at each examination, the two resulting frequency curves would have different starting points and also different shapes.

The low activity curve in Figure 22 can be transformed to the same shape and position on the $\mathrm{CV}$ axis as that corresponding to the higher activity. This is done by multiplying the $\mathrm{CV}$ values of the frequency curve for the lower activity concentration by the ratio of the mode values of the respective frequency curves (Eq 1).

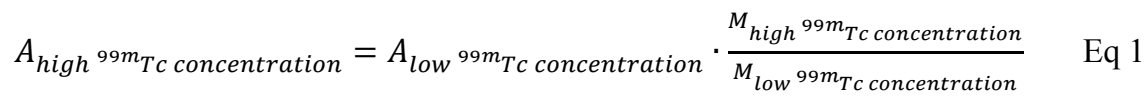

where $\mathrm{A}$ is the vector of $\mathrm{CV}$ values generating the frequency curve and $\mathrm{M}$ is the mode value. The indices "high" and "low" indicate the level of activity concentration.

In more complex distributions, as in human lungs, there are both heterogeneities in the activity distribution and the periphery effect. The frequency curves are therefore not as bell-shaped as in Figure 22. As illustrated in Figure 23, at high activity the curves are asymmetric, with a distinct peak at low CV values (corresponding to the healthy regions of the lung) and a tail at higher CV values (corresponding to the heterogeneities and the periphery). By reducing the activity, the frequency curve becomes a symmetric bell. This change in shape is caused by the gradual "loss of information" by the SPECT system due to the increase in statistical noise. 


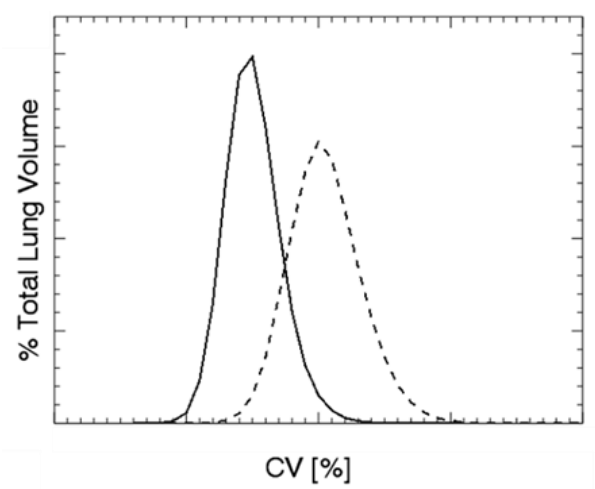

Figure 22. Frequency curves based on the uniform distribution 1 in Table 1, for two different activity levels. The curve to the right (dashed line) is based on 50\% of the activity compared to the curve to the left (solid line). A three-voxel wide layer was eroded from the periphery before analysis resulting in almost complete exclusion of the periphery effect.

If Eq 1 were applied to transform the frequency curve based on a low to a higher average particle concentration, it would not fully succeed. This is due to that the tail end, containing the heterogeneity information, cannot be restored (see Figure 23); the transformed CV curve would look like that of a uniform activity distribution. Furthermore, when transforming a frequency curve based on a high to a lower average particle concentration, the resulting frequency curve would contain too long a tail of CV values. Therefore, any transformation has to be performed with caution. In the presented compensation method the transformation of the shape of the frequency curves was therefore performed according to Eq 2 that result in less pronounced changes in the shape of the frequency curve compared to using Eq 1.

$A_{\text {compensated }}=A_{\text {shifted }} \cdot \frac{9.5}{9.5-\text { shift }}$

where $\mathrm{A}_{\text {compensated }}$ is the vector of $\mathrm{CV}$ values generating the compensated frequency curve and $\mathrm{A}_{\text {shifted }}$ is the shifted $\mathrm{CV}$ vector. The mode value of the mean curve defined in the Results section of paper IV is $9.5 \%$. The shift is negative if the subject has a lower activity concentration in its healthy regions compared to the norm. Applying a shift of a frequency curve is performed by adding the shift value to all elements in the CV vector. As shown in Eqs 1 and 2, a change in shape is performed by multiplying the elements in the $\mathrm{CV}$ vector by a value. 


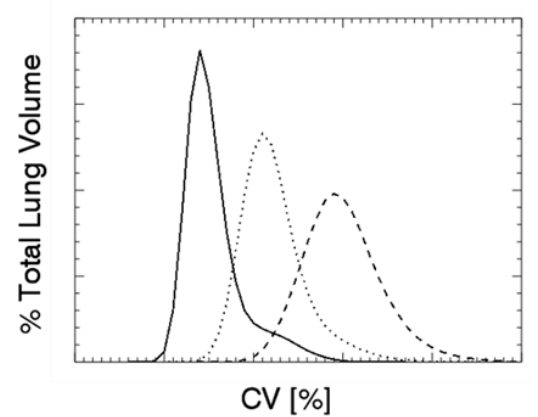

Figure 23. The change in frequency curve due to different average concentrations of ${ }^{99 m}$ Tc particles in a phantom lung with heterogeneities. Mean frequency curves based on 3 decreasing average number of ${ }^{99 m}$ Tc particles per unit volume are shown, with the highest (left solid line), middle (centre dotted line) and the lowest (right dashed line). The mean curves are based on 20 noise realisations. (from Appendix paper IV)

The effect of the compensation can in some cases be too large, even though we have chosen a compensation method that has a lower impact on the frequency curve compared to that using Eq 1. Therefore there is a limiting minimum activity concentration for which the compensation method is useful. This is illustrated in Figure 24b. When the proposed compensation method was applied to the frequency function of this subject, the resulting AUC ( $\mathrm{CV}>20 \%)$ value was even lower than that for the subjects representing the mean curve (healthy, tall and younger). This is a misleading result. Therefore this subject was excluded from the study. Patient P1 had a shift as large as the excluded healthy subject (Figure 24a). When the proposed compensation method was applied to the frequency function of this patient, the resulting AUC (CV>20\%) value was not lower than that for the subjects representing the mean curve. This patient was therefore not excluded. The explanation to why the frequency curve of P1 did not drop under the mean curve for CV values greater than $20 \%$ can be explained by disease related heterogeneities in the activity distribution. The interpretation of the compensated frequency curve of patient P1 would therefore be that P1 is found more healthy than he/she really is. Exclusion might instead be based on the size of the shift and not on the resulting $\mathrm{AUC}(\mathrm{CV}>20 \%)$ value as done in paper IV. 

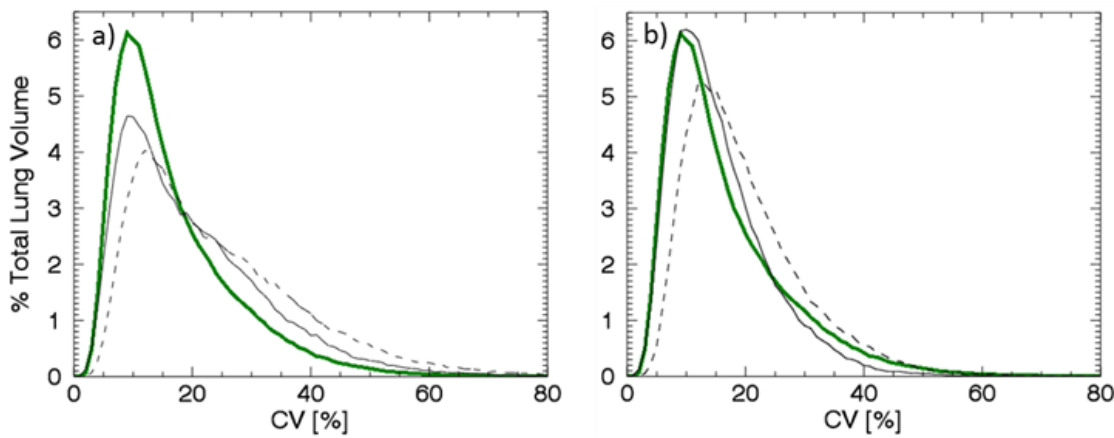

Figure 24. Frequency curves before and after compensation for a) patient P1 b) the excluded subject in paper IV. For both subjects, curves are shown for before (black dashed line), after (black solid line) compensation, together with the compensated mean curve (subjects S1-S4)(green solid line). The healthy subject in b) was excluded due to a relatively low concentration of Tc particles per unit volume in the lung.

\section{The parametric image and sub-volumes}

When the cubic kernel steps through the reconstructed volume the calculated CV values are stored in a new matrix, the CV matrix or parametric image (to the right in Figure 25). The image has the same matrix size as the reconstruction. The middle position of the kernel defines the position of the $\mathrm{CV}$ value in the parametric image. The image can be presented as desired using for example various colour tables.

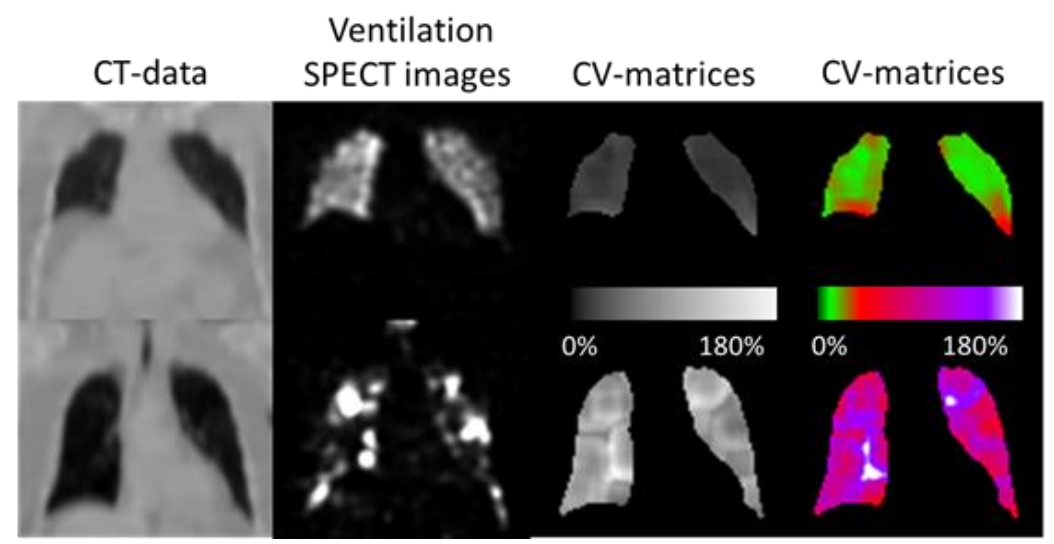

Figure 25. Coronal slices of two subjects from paper II. Top row shows a healthy volunteer and bottom row a COPD patient. Notice the patchy gas distribution in the SPECT image and resulting high CV values for the patient compared to the healthy volunteer. The two columns to the right are based on the same information but visualised using different colour tables. (Image from poster ii) 
To illustrate where different CV values are generally positioned in the lung, we show CV values below $9.5 \%$ as green, between $9.5 \%$ and $20 \%$ as yellow and above 20\% in red, for the subjects S1 and S12 in paper IV (Figure 26). Lung function tests for both subjects where interpreted as normal, but subject S12 exhibited possible early signs of emphysema. Subject S1 showed a lower AUC $(C V>20 \%)$ compared to subject S12. Areas in green and yellow are in the peak of the mean curve and are found in the centre of the lung for both subjects. Due to the moderate spatial resolution of the gamma camera system, (false) high $\mathrm{CV}$ values are generated in the periphery of the lung of a healthy subject (the periphery effect (Norberg et al., 2013)). Therefore, red areas are found in the periphery of the lungs of both subjects, but a larger proportion is found in subject S12 compared to subject $\mathrm{S} 1$.

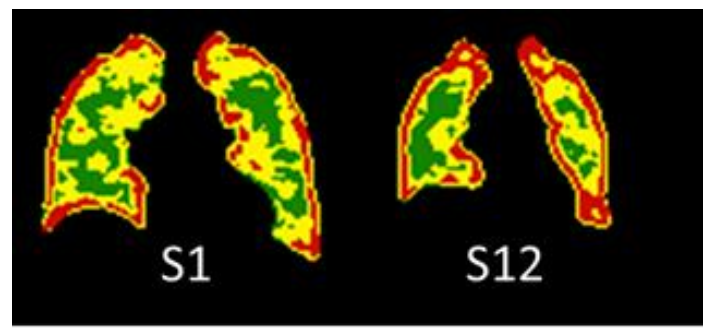

Figure 26. Coronal slices of the parametric images for subjects $S 1$ and $S 12$ in paper IV. CV slices with CV values below 9.5\% in green, between $9.5 \%$ and $20 \%$ in yellow and above $20 \%$ in red for subject $S 1$ to the left and subject $S 12$ to the right.

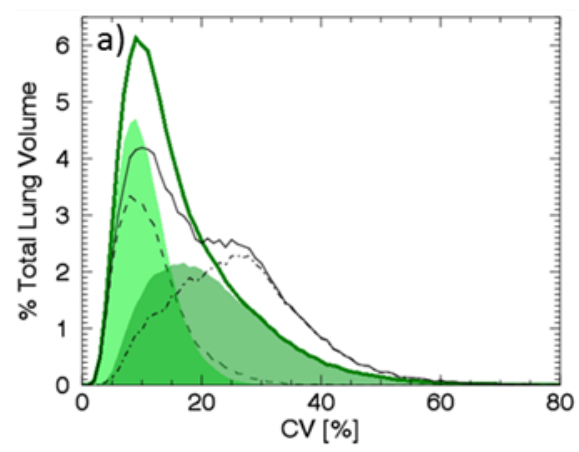

b)

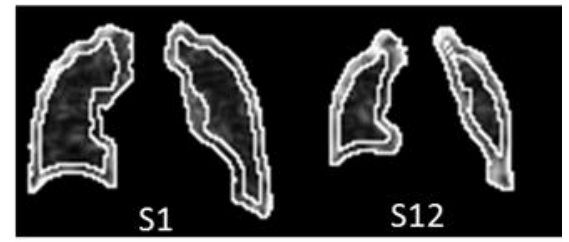

Figure 27. Entire, inner, peripheral frequency curves for mean (S1-S4) and S12, and coronal inner/ peripheral contours (paper IV). The segmented lung divided into inner and outer volumes, a) the mean frequency curve (S1-S4) (solid green line), and its inner (light green area), and outer (dark green area) volume components, as well as the S12 frequency curve (solid black line), and its inner (black dashed line) and outer (black dash-dotted line) volume components. b) Coronal slices of the CV matrix for S1 (left, used to calculate the mean) and S12 (right). Contours indicate the inner and outer volumes. 
The CV values for one subject can be divided in sub-groups depending on which sub-volumes they correspond to. Frequency curves for each sub-volume can then be constructed. To illustrate this, an inner and outer volume was created for S12. The inner volume of interest was created by eroding the outer boundary of the segmented lung by three voxels. The outer volume of interest was created by subtracting the inner volume from the original, segmented lung. The frequency curves of subject S12 are shown in Figure 27. For comparison, the same procedure was applied to the mean frequency curve for subjects S1-S4 from paper IV (Figure 27). The mean curve includes the four subjects (S1-S4) with the lowest $\mathrm{AUC}(\mathrm{CV}>20 \%)$ values of the 13 examined healthy subjects in that study. The main difference in the CV distributions of the mean curve and S12 is a shift of $\mathrm{CV}$ values in the outer volume, but not in the inner volume. This is consistent with the observations made studying the parametric images in Figure 26. 


\section{EVALUATION OF LUNG FUNCTION ON HUMAN SUBJECTS}

The ability of the method was evaluated using healthy volunteers and patients with severe COPD (paper II). Beforehand, this was an expected easy task for the method. Nevertheless, some difficulties were discovered when applying the method in a clinical setting such as, estimation of inhaled amount of Technegas at the time of examination, and varying starting points of the frequency curves between subjects. The compensation method was therefore developed, aiming to handle this problem. Finally, the ability of the method, including the compensation method, was tested in a small pilot study using healthy volunteers (paper IV).

\section{Using humans with severe COPD}

The ability of the $\mathrm{CV}_{\mathrm{T}}$ method to discriminate between five healthy volunteers and five patients with advanced COPD was evaluated (paper II).

\section{Material and Methods}

The patient group consisted of five COPD patients with grade 3 or grade 4 according to the GOLD standard. The group of healthy volunteers consisted of five healthy $\left(\mathrm{FEV}_{1}>80 \%\right.$ predicted) non-smokers (who never smoked), older than 40 years (H1-H5).

Starting from residual capacity, subjects inhaled a deep breath of Technegas and hold their breath for 2 to $5 \mathrm{~s}$ and then expired. This manoeuvre was repeated one to several times until the amount of ${ }^{99 \mathrm{~m}} \mathrm{Tc}$ deposited in the lungs was estimated to be approximately $75 \mathrm{MBq}$. The estimation was based on the count level in two opposite planar projections (left and right). The subjects inhaled the gas in a supine position immediately before the ventilation SPECT acquisition in the same position (Figure 3 ). The ventilation acquisition was followed by a low-dose CT examination performed using the X-ray source and detector mounted on the gamma camera (Hawkeye, GE Infinia, Milwaukee, WI, USA). Thereafter a perfusion acquisition was performed with $225 \mathrm{MBq}$ in order to give the subjects a clinical interpretation of possible ventilation/perfusion mismatch. The total effective dose for this protocol (ventilation $1.1 \mathrm{mSv}$ (ICRP-80, 1998); CT $2 \mathrm{mSv}$; and perfusion $2.7 \mathrm{mSv}$ (ICRP-53, 1988)) was estimated to $5.8 \mathrm{mSv}$, which required a total acquisition time of $40 \mathrm{~min}$ (ventilation $20 \mathrm{~min}$, CT $5 \mathrm{~min}$; and perfusion 15 min). The subjects were instructed to breathe normally during SPECT and CT acquisition and to lie still, without moving in-between acquisitions. The data was acquired for both ventilation and perfusion using a double-headed gamma camera 
(GE Infinia, Milwaukee, WI, USA) with a low energy high resolution (LEHR) collimator and 120 projections.

Reconstructions were performed using OSEM including attenuation, scatter and CDR compensation. Ten iterations and 16 subsets were used, i.e. 160 updates. The side length of the cubic voxels in the reconstructed image was 3.45 $\mathrm{mm}$. The reconstructed images were post-filtered with a Butterworth filter with a cut-off frequency of $0.5 \mathrm{~cm}^{-1}$ and a power of 6 .

To define the extension of the lung, a semi-automated procedure was used for each subject. A one-voxel-wide layer of the lung was eroded from the periphery of the segmented lung before analysis. The cubic kernel used was $3 \times 3 \times 3$ $\mathrm{cm}^{3}$ (i.e. $9 \times 9 \times 9$ voxels). As threshold value, the mode value of the mean frequency curve based on the activity distributions of the five healthy volunteers was used. AUC $\left(\mathrm{CV}_{\mathrm{T}}\right)$ was calculated for all activity distributions. The MannWhitney $U$ test was performed comparing the five COPD patients with the five healthy volunteers.

\section{Results and discussion}

The study showed that our way of performing lung SPECT and calculating the AUC $\left(\mathrm{CV}_{\mathrm{T}}\right)$ significantly discriminates non-smoking healthy volunteers from patients with severe COPD $(\mathrm{p}<0.05)$ (Figure 28).

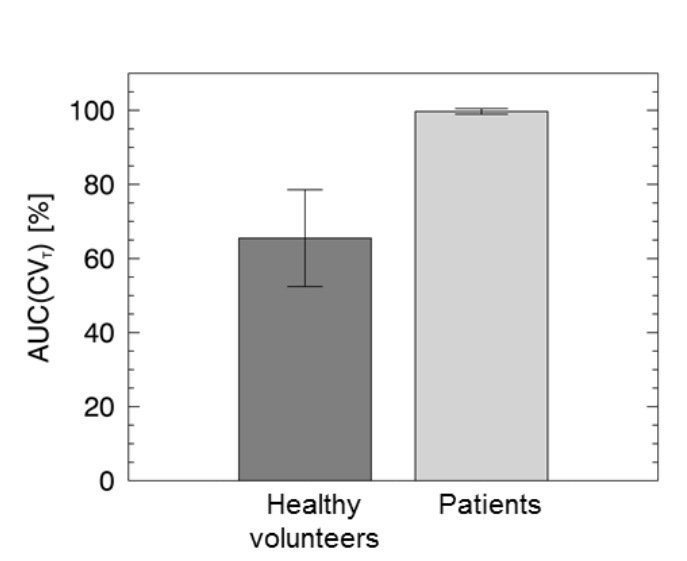

Figure 28. The mean $A U C\left(C V_{T}\right)$ for the human in vivo study with error bars representing the $95 \% \mathrm{CI}$.

This result was based on the frequency curves in Figure 14. The threshold value for the human study of $22 \%$ was somewhat greater than for the phantom study, $20.5 \%$. This might be due to the shape of the bronchial tree and the gravity influencing the human lung with the subject in supine position. 
A $95 \% \mathrm{CI}$ of the $\mathrm{AUC}\left(\mathrm{CV}_{\mathrm{T}}\right)$ value of the healthy volunteers of $\pm 13 \%$ around the mean was found. This large variation may be explained by the genetic variations, age effects, and different histories of occupational and environmental exposures of noxious particles and gases, e.g. passive exposure to tobacco smoke. Furthermore, small shifts between healthy subjects, along the CV axis of the frequency curves were present (Figure $14 \mathrm{~b}$ ). This thought to be due to the amount of inhaled Technegas in relation to the volume it was distributed in, which also contributes to the large variation in $\mathrm{AUC}\left(\mathrm{CV}_{\mathrm{T}}\right)$.

It was found difficult to estimate the amount of inhaled Technegas at the time of examination. This was due to fluctuating values (counts per second) during readout of the two opposite planar projections and a constant factor (25 $\mathrm{MBq} / \mathrm{kcps}$ ) for all subjects. The inhaled activity was also estimated after examination using the number of counts in ventilation and perfusion projections together with the known administered perfusion activity. The latter estimation was assumed to be the most correct. At the time of examination, the activity was generally slightly overestimated (Figure 29). The estimation of inhaled activity after examination showed a variation between the subjects from approximately 50 to $150 \mathrm{MBq}$ with a mean at $90 \mathrm{MBq}$ (corresponding to $1.3 \mathrm{mSv}$ ). On average the total number of counts in the ventilation projections for a subject was $4.8 \times 10^{6}$, resulting in a sensitivity of $22 \mathrm{cps} / \mathrm{MBq}$. This is $30 \%$ more counts compared to the simulation study in paper II, and an equivalent sensitivity factor.

It was also found difficult to pinpoint a predetermined activity. Some subjects inhaled excessively much at the first breath while other needed several breaths.

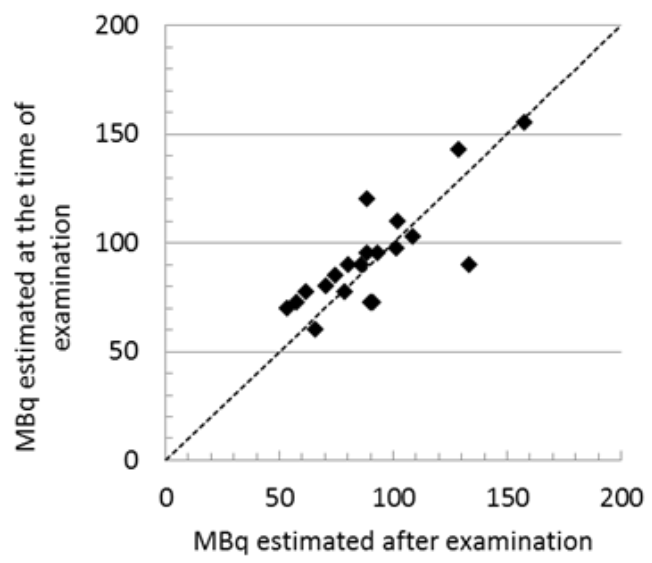

Figure 29. Inhaled activity estimated at the time of examination and after examination. The latter was assumed to be the most correct. All subjects included in the thesis are plotted. 
The frequency curves in Figure 14, indicate that a greater threshold value than the mode value would result in a greater separation between healthy and diseased subjects in Figure 28.

\section{Using healthy humans}

In this study we introduced the compensation method, that compensates for subject-to-subject variations of activity uptake, making the CV values from healthy lung volumes of different subjects comparable (paper IV). This adaptation made it possible to discriminate between subjects with less pronounced differences in ventilation heterogeneity using the $\mathrm{CV}_{\mathrm{T}}$-method. The potential advantages of quantitative heterogeneity measurements were explored by performing a pilot study with healthy human subjects, i.e. those without documented lung disease, respiratory symptoms or lung function abnormalities. The outcome was discussed in relation to gender, age, size of the lung, subtle findings on lung function tests, reported allergies, and smoking history.

\section{Material and Methods}

Fourteen human subjects were included who were without documented lung disease or respiratory symptoms. The 14 subjects were examined by lung SPECT and lung function tests. The subjects were also asked to fill in a health questionnaire. The questionnaire covered for example pulmonary symptoms, smoking history, allergies, hypersensitivity and medications. In addition, two patients with documented airway disease or pulmonary symptoms underwent the same examination procedure (referred to as P1 and P2). P1 suffered from asthma and homozygote alfa-1-antitrypsin deficiency and P2 had advanced COPD with emphysema. Gender, age, height, weight, body mass index (BMI), smoking status and history of allergy for these 16 subjects are presented in Tables 5 .

The acquisition, reconstruction and analysis were performed as in the human study in paper II (using humans with severe COPD) besides a few exceptions. We decided to include the potential information from the lung periphery in the analysis even though, including the whole lung in the analysis, makes it more difficult to distinguish between uniform and less uniform distributions. Based on the results from the optimisation study in paper III, four iterations and 16 subsets were used, i.e. 64 updates, together with a cut-off frequency of $0.6 \mathrm{~cm}^{-1}$ of the Butterworth filter and a $1 \times 1 \times 1 \mathrm{~cm}^{3}$ (i.e. $3 \times 3 \times 3$ voxels) kernel.

The variation of activity uptake between subjects was compensated for in the calculation of $\mathrm{CV}$ values, based on the assumption that each subject had a healthy lung volume and that the healthy volumes in all subjects should generate equivalent $\mathrm{CV}$ values (for further details see the section "The compensation method", page 49-53). As threshold value, both the mode value of healthy subjects $(\mathrm{CV}=9.5 \%)$, and an increased value $(\mathrm{CV}=20 \%)$ were used. 
Table 5. Characteristics of the subjects in order of increasing $\mathrm{AUC}(\mathrm{CV}>20 \%)$ value.

\begin{tabular}{|c|c|c|c|c|c|c|c|c|c|c|c|c|}
\hline 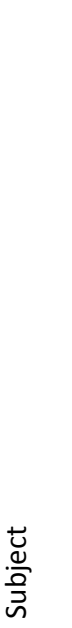 & 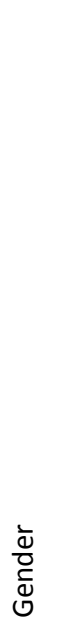 & 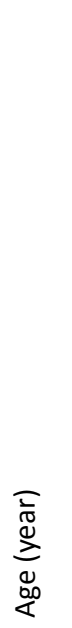 & 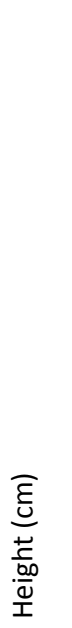 & 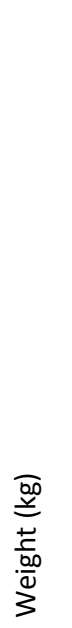 & 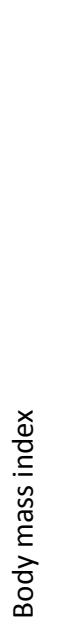 & 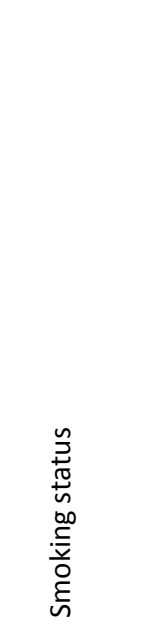 & 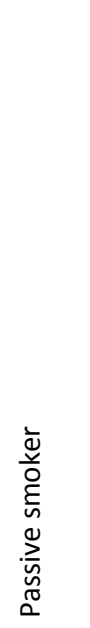 & 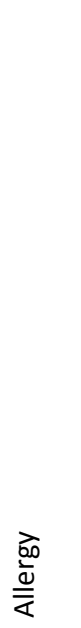 & 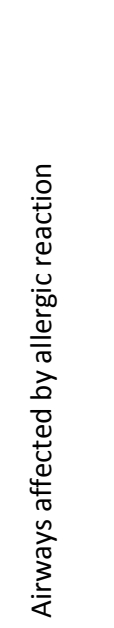 & 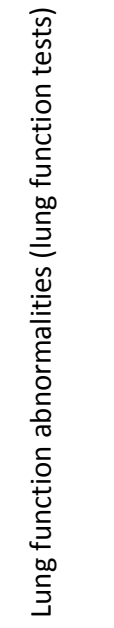 & 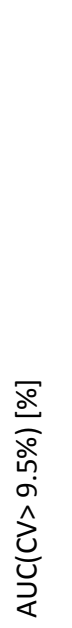 & 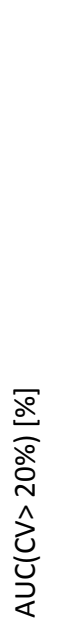 \\
\hline S1 & $M$ & 49 & 191 & 82 & 22.5 & Never & No & Yes $^{a}$ & Upperi & $\mathrm{No}^{1}$ & 76 & 29 \\
\hline S2 & $M$ & 49 & 185 & 77 & 22.5 & Never & No & $Y_{e s}^{b}$ & Upper ${ }^{i}$ & No & 77 & 30 \\
\hline S3 & $M$ & 50 & 190 & 85 & 23.5 & Never & No & $Y_{e s}^{c}$ & Upper ${ }^{i}$ & No & 78 & 30 \\
\hline S4 & $M$ & 48 & 187 & 75 & 21.4 & Never & Yes' $^{\prime}$ & No & - & No & 77 & 31 \\
\hline S5 & $M$ & 50 & 178 & 72 & 22.7 & Never & No & No & - & No & 78 & 33 \\
\hline S6 & $M$ & 69 & 181 & 100 & 30.5 & Never & Yes $^{\prime}$ & No & - & No & 78 & 34 \\
\hline S7 & $M$ & 60 & 188 & 85 & 24.0 & Current & - & No & - & $\mathrm{No}^{2}$ & 78 & 34 \\
\hline S8 & $\mathrm{F}$ & 65 & 177 & 66 & 21.1 & Current & - & $Y_{e s}^{d}$ & Loweri & No & 80 & 34 \\
\hline S9 & $F$ & 51 & 170 & 85 & 29.4 & Never & Yes $^{\prime}$ & No & - & No & 78 & 36 \\
\hline S10 & $M$ & 75 & 175 & 92 & 30.0 & Never & No & $Y_{e s}{ }^{e}$ & Upper ${ }^{i}$ & $\mathrm{No}^{3}$ & 79 & 39 \\
\hline S11 & $M$ & 73 & 175 & 77 & 24.9 & Ex-smoker & - & No & - & No & 80 & 41 \\
\hline S12 & $\mathrm{F}$ & 67 & 172 & 78 & 26.4 & Never & Yes" & No & - & $\mathrm{No}^{+}$ & 83 & 47 \\
\hline S13 & $\mathrm{F}$ & 67 & 167 & 79 & 28.3 & Never & Yes "'I & Yes $^{f}$ & Lower ${ }^{\mathrm{iii}}$ & $\mathrm{No}^{4}$ & 84 & 47 \\
\hline P1 & $M$ & 55 & 176 & 85 & 27.4 & Never & - & - & - & $\mathrm{Yes}^{3,5+t}$ & 83 & 43 \\
\hline P2 & M & 81 & 167 & 58 & 20.8 & Ex-smoker & - & - & - & Yes & 90 & 61 \\
\hline
\end{tabular}




\section{Notation index to Table 5}

${ }^{I} 20$ years during childhood

${ }^{I I} 14$ years during childhood and occupational

III 10 years occupational

${ }^{a}$ grass, pollen and dust

${ }^{b}$ fur animals and grass

${ }^{c}$ fur animals and pollen

${ }^{d}$ strong fragrances

${ }^{e}$ cat and chocolate

${ }^{f}$ birch pollen, timothy, grass, nuts, penicillin and sulfonamide

${ }^{i}$ rhinoconjunctivitis

${ }^{i i}$ cough

${ }^{i i i}$ shortness of breath

${ }^{1}$ subject medicated with nasal spray of Budesonid at time of examination

${ }^{2}$ based on $F E V_{1}, F V C, F E V_{1} / F V C, T L C$ and $R V$ without bronchodilation

${ }^{3}$ based on DLCOc before and FEV $, F V C, F E V_{1} / F V C$, TLC and RV after bronchodilation

${ }^{4}$ based on $F E V_{1}, F V C$ and $F E V_{1} / F V C$ without bronchodilation

${ }^{5}$ subject medicated with inhalation of Budesonid and Formoterol in combination at time of examination

${ }^{\dagger}$ possible early signs of emphysema based on a reduced DLCOc value (see Table 4) and an elevated haemoglobin concentration in the blood

${ }^{t+}$ moderate obstruction and signs of reversibility

Linear regression analysis was used to determine associations between AUC $(C V>20 \%)$ and the explanatory variables age, height, BMI, smoking status and allergy, respectively. Linear correlations were also determined in the same way for $\mathrm{AUC}(\mathrm{CV}>20 \%)$ and each of the lung function values recorded in healthy subjects: RV, RV/TLC, and DLCOc. Lung function values recorded without previous bronchodilation and expressed in non-normalised values, i.e. not \% predicted, were used to compare with $\mathrm{AUC}(\mathrm{CV}>20 \%)$ data, since lung SPECT examinations were performed and expressed accordingly. Furthermore, the relationship between lung function abnormalities (yes or no, according to interpreted lung function tests) and the $\mathrm{AUC}(\mathrm{CV}>20 \%)$ value was assessed in the same manner, with the difference that in this case, both healthy subjects and patients were included. 


\section{Results and discussion}

We expected subjects with gradually less altered lung function to generate gradually lower $\mathrm{AUC}(\mathrm{CV}>20 \%)$ values. Therefore, we found the result of this pilot study very promising, since the list of subjects placed in order of increasing AUC $(\mathrm{CV}>20 \%)$ value (Table 5), calculated incorporating the compensation method, supported this idea. We showed that patients (P1 and P2) generated significant higher AUC (CV $>20 \%)$ values compared to healthy subjects (S1-S13) $(p=0.006)$, which is consistent with the result of the previous human study in paper II. Despite the low sample size in this pilot study, a group of subjects was identified who never smoked, and were of the same gender (male), age bracket (48-50 years) and physique (height $185-191 \mathrm{~cm}$ and weight $75-85 \mathrm{~kg}$ ), male subjects S1-S4 (Table 5). These subjects displayed the lowest AUC $(\mathrm{CV}>20 \%)$ values. A mean frequency curve was constructed based on these subjects (hereafter called the mean curve) (Figure 30a). Based on the mean curve and the $\mathrm{CV}_{\mathrm{T}^{-}}$
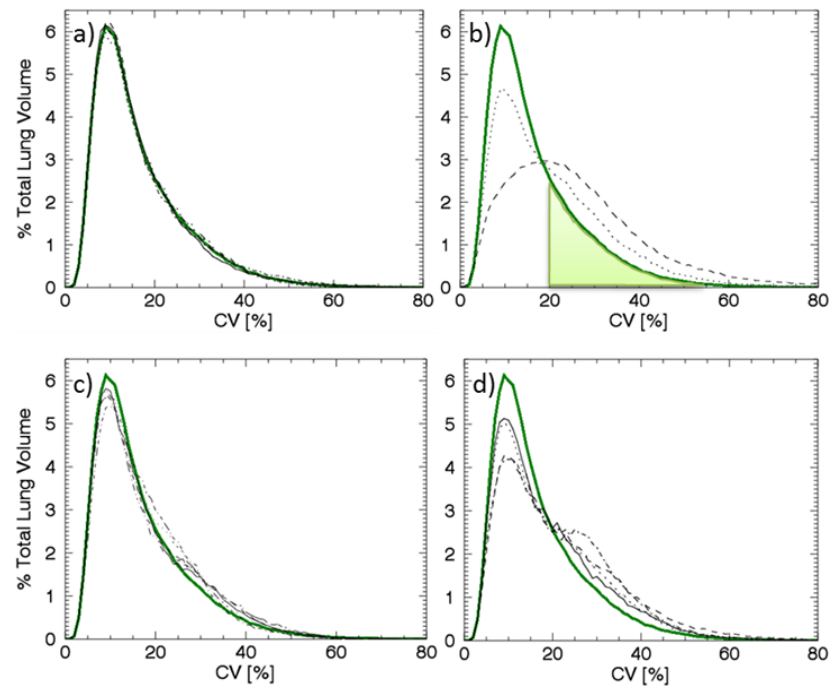

Figure 30. Frequency curves for low-AUC(CV>20\%) subjects, patients (P1-P2), intermediate-AUC(CV>20\%) subjects (S5-S9), and high-AUC(CV>20\%) subjects (S10S13), with mean (S1-S4). a) Frequency curves corresponding to subject; S1 (solid line); $S 2$ (dotted line); S3 (dashed line); and S4 (dashed-dotted line), together with their constructed mean curve in green. b) Frequency curves of patient; $P 1$ (dotted line); and P2 (dashed line). AUC for CV values greater than $20 \%$, i.e. AUC(CV>20\%), for the mean curve is marked as light green. c) Five frequency curves corresponding to intermediate $A U C(C V>20 \%)$ values of subject; S5 (solid line); S6 (dotted line); S7 (dashed line); S8 (dashed-dotted line); and $S 9$ (dashed-dotted-dotted line). d) Four frequency curves corresponding to the highest $A U C(C V>20 \%)$ values of subjects; subject S10 (solid line); S11 (dotted line); S12 (dashed-dotted line); and S13 (dashed line). All figures include the mean curve (green line). 
method from earlier studies, we defined $\mathrm{CV}_{\mathrm{T}}=9.5 \%$ as the mode value of the mean curve, and calculated and listed the AUC $(\mathrm{CV}>9.5 \%)$ for all subjects and patients in Table 5. The frequency curves of the patients P1 and P2 are substantially different from the mean curve, as shown in Figure 30b, especially for patient P2, with severe COPD. Based on Figure 30b, an additional threshold value was defined, $\mathrm{CV}_{\mathrm{T}}=20 \%$, approximately at the point where the patient curves and the mean curve intersect. Since $\mathrm{AUC}(\mathrm{CV}>20 \%)$ resulted in a favourably larger range $(29 \%-47 \%)$ compared to the range of $\mathrm{AUC}(\mathrm{CV}>9.5 \%)$ values $(76 \%-84 \%)$ for the included healthy subjects, $\mathrm{CV}_{\mathrm{T}}=20 \%$ was used hereafter. Listing the subjects in order of increasing $\mathrm{AUC}(\mathrm{CV}>9.5 \%)$ value would introduce shifts between adjacent subjects in Table 5, which implies the chosen threshold has only a minor effect on their order. The remaining healthy subjects were divided into two groups, S5-S9 and S10-13, depending on their frequency curves, which are shown in Figures 30c and 30d, respectively. The frequency curves of subjects S5-S9 exhibited small differences from the mean curve. In contrast, subjects S10S13 showed deviating frequency curves compared to the mean curve, and consequently higher $\mathrm{AUC}(\mathrm{CV}>20 \%)$ values, without abnormalities detected by lung function tests. This implies that it could be possible to detect ventilation heterogeneities by the $\mathrm{AUC}\left(\mathrm{CV}_{\mathrm{T}}\right)$ method earlier in a disease process than by lung function tests. The fact that one of the 13 healthy subjects, who exhibited possible early signs of emphysema (S12), also showed higher $\mathrm{AUC}(\mathrm{CV}>20 \%)$, tends to support the notion that the $\mathrm{AUC}\left(\mathrm{CV}_{\mathrm{T}}\right)$ method is more sensitive to minor pulmonary abnormalities than conventional lung function tests.

The proposed method confirms the expected increase in $\mathrm{AUC}(\mathrm{CV}>20 \%)$ with age $(\mathrm{r}=0.72)$. This study also demonstrated the expected decrease of AUC $(C V>20 \%)$ values with increasing height $(r=-0.82)$. The correlations were statistically significant between $\mathrm{AUC}(\mathrm{CV}>20 \%)$ and both height $(\mathrm{p}=0.001)$ and age $(p=0.006)$. We found a strong correlation between $\mathrm{AUC}(\mathrm{CV}>20 \%)$ and $\mathrm{RV} / \mathrm{TLC}(\mathrm{r}=0.74)$, as well as between AUC $(\mathrm{CV}>20 \%)$ and DLCOc $(\mathrm{r}=-0.77)$, also with high statistical significance $(\mathrm{p}=0.009)$. This supports the value of measuring heterogeneities in ventilation lung SPECT images, since these lung function parameters measure the volume with closed airways and air trapping (RV/TLC) and reflects the amount of ventilation/perfusion mismatch in the lungs (DLCOc). One might argue that the strong correlation found between the AUC $(C V>20 \%)$ value and the lung function values RV/TLC and DLCOc would contradict the value of lung SPECT measurements in comparison to lung function testing. However in this context, it is important to note that all except one of the subjects (S12) was considered fully 'normal' by a physician. Furthermore, lung SPECT provides the possibility to visualise the location of the malfunctioning volumes in the lungs (see Figure 26 and 27 in section "The parametric image and sub-volumes", page 54-56), unlike lung function tests.

In this small pilot study, no statistically significant differences were found between subjects who never smoked, current smokers and ex-smokers. However, 
we found a tendency of ex-smokers to have a higher $\mathrm{AUC}(\mathrm{CV}>20 \%)$ value than subjects who never smoked and current smokers. No difference between healthy subjects with or without stated allergies was observed. In a sub-group analysis among subjects with allergies, a notable tendency of higher AUC(CV>20\%) values was found for subjects with symptoms from the lower respiratory tract compared to the subjects with symptoms from the upper respiratory tract. This study found no BMI or RV dependence.

The main limitation of this study is the low number of participants, and consequently a single observation had a large impact on the linear correlation. With a larger number of subjects with different smoking histories and subjects with allergy symptoms from both the upper and lower respiratory tract, the tendencies found in this pilot study might find statistical significance.

Information of possible air trapping and emphysema from HRCT images of the healthy subjects in paper IV could have been beneficial, but due to the comparably high effective dose of $5.3 \mathrm{mSv}$ from this procedure such images were not taken. 



\section{REVIEW OF PUBLICATIONS}

This chapter gives a brief review of the four papers that the thesis is based upon.

\section{Paper I}

A comparison of the two reconstruction algorithms FBP and OSEM was performed using trade-off plots of the image quality parameters contrast, noise and resolution. Monte Carlo simulated lung-SPECT images of activity distributions in an anthropomorphic thorax voxel phantom were used. Parameters studied were iteration number and subsets for OSEM, collimator, noise reduction filter and associated cut-off frequencies, and activity level.

The study showed that OSEM is the preferred reconstruction method since OSEM obtains higher resolution at a lower noise level compared to FBP. A large number of iterations is needed, the LEHR is preferable, and the cut-off frequency is essential but the number of subsets and type of filter are not.

\section{Paper II}

The $\mathrm{CV}_{\mathrm{T}}$-method was presented and evaluated on reconstructions based on activity distributions in a dynamic anthropomorphic thorax phantom and on humans.

The phantom activity distributions used aimed to mimic a healthy lung and several COPD lungs with various severities. The $\mathrm{CV}_{\mathrm{T}}$-method was able to differentiate between the "healthy" lung and the "mild COPD" lung $(\mathrm{p}<0.001)$. The "mild COPD" lung had only $5 \%$ total reduction of ventilation. This difference was not visible to the human eye in the reconstructed images.

Five patients with advanced COPD and five healthy volunteers (neversmokers) performed a lung ventilation SPECT examination each. The $\mathrm{CV}_{\mathrm{T}^{-}}$ method clearly discriminated between these two groups $(\mathrm{p}<0.05)$. A large variation of the $\mathrm{AUC}\left(\mathrm{CV}_{\mathrm{T}}\right)$ value of the healthy volunteers around the mean was found. This large variation might be due to genetic variations, age effects, and different histories of occupational and environmental exposures of noxious particles and gases e.g. passive exposure to tobacco smoke. Furthermore, the variation might also be due to the amount of inhaled Technegas in relation to the lung volume it was distributed in. The threshold value used was the mode value of the mean frequency curve of the healthy subjects. Another observation made, was that a larger threshold value, about where the mean frequency curve intersects with the patients' curve, would have resulted in an even larger separation between the two groups. 


\section{Paper III}

The acquisition, reconstruction and analysis parameters were optimised to provide the best chance for the $\mathrm{CV}_{\mathrm{T}}$-method to successfully differentiate between Monte Carlo simulated lung-SPECT images of a "healthy" phantom lung and the "mild COPD" phantom lung. In paper II the "mild COPD" distribution was found to be the most difficult one to separate from the "healthy" distribution, and therefore chosen. Parameters studied were activity level, collimator, number of OSEM updates, cut-off frequency of the noise reduction filter, volume of analysis and size of the cubic kernel. Comparison was made between the resulting $\mathrm{AUC}\left(\mathrm{CV}_{\mathrm{T}}\right)$ values of the two distributions using the Mann-Whitney U-test. The combination of parameter values that resulted in the lowest $p$-value was set to be the optimal values.

The study gave two results, one when the whole lung was included in the analysis, and one when a periphery layer was excluded. The greatest separation was achieved by using a total of at least $3.64 \times 10^{6}$ counts in the projections, employing an LEHR collimator together with a Butterworth power 6 noisereduction filter with a cut-off frequency of $0.6-0.7 \mathrm{~cm}^{-1}$. When the whole lung was analysed, 64 reconstruction updates and a kernel with a side length of $1.0 \mathrm{~cm}$ gave the best result. For the reduced lung volume a greater number of updates and a larger kernel size are needed.

\section{Paper IV}

An adaptation was introduced that compensates for subject-to-subject variation of activity uptake, identified in paper II, making the CV values from healthy lung volumes of different subjects comparable. This adaptation makes it possible to better evaluate the ability of the $\mathrm{CV}_{\mathrm{T}}$-method to discriminate between subjects with less pronounced differences in ventilation heterogeneity. The potential advantages of quantitative heterogeneity measurements were explored by performing a pilot study with 14 healthy human subjects and two patients. All subjects performed a lung ventilation SPECT examination, lung function tests and filled in a health questionnaire about e.g. pulmonary symptoms, smoking history, allergies and medications. The threshold value used in this paper shifted from the mode value $(9.5 \%)$ of a mean healthy frequency curve to $(20 \%)$ where this curve intersects with the patients' curve.

Patients with lung function abnormalities, according to lung function tests, generated higher $\mathrm{AUC}(\mathrm{CV}>20 \%)$ values compared to healthy subjects $(\mathrm{p}=0.006)$. Strong linear correlations with the AUC(CV>20\%) values were found for age $(p=0.006)$ and height $(p=0.001)$. These results demonstrated that ventilation heterogeneities increased with age and that they depend on lung size. Strong linear correlations were found for the lung function value related to indices of airway closure/air trapping, RV/TLC ( $\mathrm{p}=0.009)$, and DLCOc $(\mathrm{p}=0.009)$, a value partly 
related to supposed ventilation /perfusion mismatch. These findings support the association between conventional lung function tests and the AUC(CV>20\%) value. A tendency of ex-smokers to have a higher $\mathrm{AUC}(\mathrm{CV}>20 \%)$ value than subjects who never smoked and current smokers was found. In a sub-group analysis among subjects with allergies, a tendency of higher $\mathrm{AUC}(\mathrm{CV}>20 \%)$ values was found for subjects with symptoms from the lower respiratory tract compared to subjects with symptoms from the upper respiratory tract. Increased AUC $(C V>20 \%)$ values were also detected among subjects who had normal lung function tests but indications of conditions associated with ventilation disturbances. The results suggest that our present SPECT method has the capacity to identify minor lung function abnormalities earlier in a disease process than conventional lung function tests. 



\section{SUMMARY AND CONCLUSIONS}

A quantitative heterogeneity-detection method, the $\mathrm{CV}_{\mathrm{T}}$-method, for lung ventilation SPECT images has been developed. The method determines the proportion of the lung consisting of heterogeneities above a defined level. This proportion for one individual could be followed over time to evaluate disease advancement or outcome of various interventions. With access to normal values for different age and height groups, the method has the potential to identify mild lung function abnormalities, earlier in a disease process than conventional lung function tests. Individual three-dimensional parametric images can be obtained visualising regions with elevated heterogeneity.

The method has been shown to be capable of identifying simulated mild COPD in an anthropomorphic phantom, and to differentiate patients with severe COPD from healthy subjects. Identified difficulties and possible improvement resulted in a compensation technique and the change in used $\mathrm{CV}_{\mathrm{T}}$ threshold value. The compensation makes the $\mathrm{CV}$ values from healthy lung volumes of different subjects comparable. This adaptation made it possible to identify subjects who had normal lung function tests but indications of conditions associated with ventilation disturbances. The results suggest that our present SPECT method has the capacity to identify minor lung function abnormalities earlier in a disease process than conventional lung function tests.

Parameters included in acquisition of the gamma camera projection, reconstruction of the three dimensional image and noise reduction, analysis using the $\mathrm{CV}_{\mathrm{T}}$-method, and the their effects on the ability of the $\mathrm{CV}_{\mathrm{T}}$-method to discriminate between COPD disorders have been studied. First, the effect of various acquisition and reconstruction parameter values on image quality was evaluated. Thereafter, the most advantageous combination of acquisition, reconstruction and analysis parameters values was determined. This combination provided the best chance for the $\mathrm{CV}_{\mathrm{T}}$-method to successfully differentiate between a simulated "healthy" lung and a lung with only $5 \%$ total reduction of ventilation. As the threshold value used in the $\mathrm{CV}_{\mathrm{T}}$-method was changed in the last pilot study this indicates that there might be an even better combination of parameter values for this new condition.

\section{Future work and improvements}

Our positive findings have provided the motivation to extend the last pilot study to a full study with a larger number of subjects. 
The protocol used on our subjects has included both ventilation and perfusion acquisition. By abandoning the perfusion acquisition a higher activity level could be used for ventilation without increasing the effective dose to the individual. Another possibility is to maintain the original lower activity level to allow for repeated ventilation examinations of the same individual.

There is a risk that individuals inhale an excessively large amount of activity in their first breath. A reduced concentration of ${ }^{99 \mathrm{~m}} \mathrm{Tc}$ particle in the Technegas could solve this problem. Additional breaths will then be needed, which probably would make it easier to pin-point a desired activity level. Decreasing the concentration might be achieved by a small oxygen bleed via a needle pushed through near the mouthpiece. This procedure is used to reduce the risk of hypoxia especially in elderly patients (Burch, 2013).

The compensation technique has its shortcomings. Eliminating the need for it requires the frequency curve of each individual to be right positioned on the CVaxis already at the time of examination. If this was the case, each individual would obtain a more correct result from the $\mathrm{CV}_{\mathrm{T}}$-method and a comparison between individuals would become more reliable. Furthermore, no examination would be rejected due to an excessively low amount of inhaled activity. One solution could be to perform a simplified evaluation "on line" while the individual is still in the right position on the couch. This could be achieved by expanding the "test" acquisition after inhalation of Technegas from the two left-right projections to a few more, reconstruct them and calculate a frequency curve. This procedure could then be repeated until the frequency curve is properly positioned.

The results in this study were mostly based on global measures including the whole lung (or a reduced lung). In one case the lung was divided into an inner and outer volume, respectively. The resulting sub-volume frequency curves together with corresponding parametric image revealed which sub-volume contained elevated heterogeneity values. Other sub-volumes, such as lung lobes, would be of interest.

The $\mathrm{CV}_{\mathrm{T}}$-method could be adapted to positron emission tomography (PET) imaging. Ventilation examinations using PET can be performed using for example inhaled Galligas (carbon nanoparticles connected to Gallium-68) (Ament et al., 2013; Hofman et al., 2011). Advantage with PET compared to SPECT is a higher spatial resolution, full tomographic acquisition $360^{\circ}$ around the subject with potentially better regional quantification (Hofman et al., 2011). It would be very interesting if the $\mathrm{CV}_{\mathrm{T}}$-method also could be adapted to magnetic resonance imaging (MRI) using hyper polarised helium (Altes et al., 2001; Samee et al., 2003; Emami et al., 2013).

Other descriptive measures describing the shape of the frequency curve (Jobse et al., 2012; Sando et al., 1997) e.g. the mean, mean standard deviation, skewness, excess kurtosis and the maximal $\mathrm{f}(\mathrm{x})$-value might be valuable measures. 


\section{ACKNOWLEDGEMENTS}

A number of people have helped and supported me during the work with this thesis and I would now like to express my sincere gratitude to you all!

My supervisors Michael Sandborg and Gudrun Alm Carlsson for support and guidance. Gudrun has patiently improved my manuscripts, especially my latest. She really encouraged me to the bitter end.

My co-supervisor Agnetha Gustafsson for guidance and for being there through ups and downs both with this thesis and on the bicycle. "Do it again, do it right!" and "How difficult can it be?" are phrases that have been heard repeatedly.

My co-authors Lennart Persson, Birgitta Schmekel and Björn Bake for medical expertise and for letting the $\mathrm{CV}_{\mathrm{T}}$-method try its wings in the real world. One extra thanks to Lennart for his enthusiasm, creative thinking and great support, making things possible. Also thanks to Bengt Holmberg for clinical interpretation of the lung SPECT images.

My co-workers Annika Enander, Gunvor Josefsson, Helen Brundin, Julia Studeny and Majne Malmgren for carrying out all the examinations in the clinic for me. Also thanks to Anette Davidsson for valuable discussions about spirometry and life as a PhD-student.

My co-author and PhD-student friend Anna Olsson, and colleague Marcus Ressner for help, support and encouragement, which has been very important to me.

My volunteers for participating in my studies. Without you, this work would never have taken place.

Eva Lund, for encouragement and support, apples and rhubarb (the plant).

My PhD-student friends Axel, Emelie, Mattias, Magnus and Sofia for great support. Keep on working!

All my colleagues and co-workers for chats and laughter during coffee breaks and in the corridors at Radiation Physics, Nuclear medicine and Radiation therapy department. You really enrich a day at work.

My cycling friends Agnetha, Andreas, Emma, Erik, Federico, Jonas, Marcus, Sven and Tina for sharing great moments with me.

My relative Wallis Jansson, full of vitality, who wrote her own thesis in her sixties. Inspiring girl power!

My parents Rolf and Britta, brother Jens and sister Johanna, and their families for support without really knowing what I've been doing.

Lastly, I would like to give many hugs and kisses to my lovely family Noa, Teo, Loi and Leif. Finally, we will be able to spend more time together again!

And to all of you who don't find your name above, thank you too! 


\section{REFERENCES}

Altes T A, Powers P L, Knight-Scott J, Rakes G, Platts-Mills T A, de Lange E E, Alford B A, Mugler J P, 3rd and Brookeman J R 2001 Hyperpolarized $3 \mathrm{He}$ MR lung ventilation imaging in asthmatics: preliminary findings Journal of magnetic resonance imaging : JMRI 13 378-84

Ament S J, Maus S, Reber H, Buchholz H G, Bausbacher N, Brochhausen C, Graf F, Miederer M and Schreckenberger M 2013 PET lung ventilation/perfusion imaging using (68)Ga aerosol (Galligas) and (68)Galabeled macroaggregated albumin Recent results in cancer research. Fortschritte der Krebsforschung. Progres dans les recherches sur le cancer 194 395-423

Amis T, Crawford A, Davison A and Engel L 1990 Distribution of inhaled 99mtechnetium labelled ultrafine carbon particle aerosol (Technegas) in human lungs European Respiratory Journal 3 679-85

Ax M, Karlsson L L, Sanchez-Crespo A, Lindahl S G, Linnarsson D, Mure M and Petersson J 2013 Regional lung ventilation in humans during hypergravity studied with quantitative SPECT Respiratory physiology \& neurobiology 189 558-64

Bajc M, Neilly J B, Miniati M, Schuemichen C, Meignan M and Jonson B 2009a EANM guidelines for ventilation/perfusion scintigraphy : Part 1 . Pulmonary imaging with ventilation/perfusion single photon emission tomography Eur J Nucl Med Mol Imaging 36 1356-70

Bajc M, Neilly J B, Miniati M, Schuemichen C, Meignan M and Jonson B 2009b EANM guidelines for ventilation/perfusion scintigraphy : Part 2 . Algorithms and clinical considerations for diagnosis of pulmonary emboli with V/P(SPECT) and MDCT Eur J Nucl Med Mol Imaging 36 1528-38

Bake B 2000 Lungfysiologi och lungdiagnostik vid lungsjukdom, ed L Bäcklund, et al. (Sverige: Studentlitteratur)

Burch W M, Visiting Fellow, John Curtin School of Medical Research

Australian National University (1976-2008), E-mail, 30th of July 2013

Burch W M, Sullivan P J and McLaren C J 1986 Technegas--a new ventilation agent for lung scanning Nucl Med Commun 7 865-71

Chen D, Webb W R, Storto M L and Lee K N 1998 Assessment of air trapping using postexpiratory high-resolution computed tomography Journal of thoracic imaging 13 135-43

Eberl S, Chan H K and Daviskas E 2006 SPECT Imaging for Radioaerosol Deposition and Clearance Studies Journal of aerosol medicine : the official journal of the International Society for Aerosols in Medicine 19 820

Emami K, Hamedani H, Kadlecek S, Han B, Xin Y, Ishii M, Rossman M and Rizi R R 2013 Hyperpolarized gas fractional ventilation imaging study in healthy subjects, asymtomatic smokers and obstructive pulmonary disease 
patients- Perliminary results. In: AJRCCM Conference, (Pennsylvania Convention Center

Frey E C and Tsui B M W 1996 A new method for modelling the spatiallyvariant, object-dependant scatter response function in SPECT. In: IEEE Nuclear Science Symp. 1996 pp 1082-6

Cleveland Clinic, US, Pulmonary Function Testing, Last Updated 1st of August 2010, Available from:

http://www.clevelandclinicmeded.com/medicalpubs/diseasemanagement/p ulmonary/pulmonary-function-testing/\#top

From the Global Strategy for the Diagnosis, Management and Prevention of COPD, Global Initiative for Chronic Obstructive Lung Disease (GOLD) 2011, http://www.goldcopd.org/

Harris P J F and Harris P J F 2001 Carbon Nanotubes and Related Structures: New Materials for the Twenty-first Century: Cambridge University Press)

Hedenstierna G 2000 Lungfysiologi och lungdiagnostik vid lungsjukdom, ed L Bäcklund, et al. (Sverige: Studentlitteratur)

Hofman M S, Beauregard J M, Barber T W, Neels O C, Eu P and Hicks R J 2011 68Ga PET/CT ventilation-perfusion imaging for pulmonary embolism: a pilot study with comparison to conventional scintigraphy $J \mathrm{Nucl}$ Med $\mathbf{5 2}$ 1513-9

Hudson H M and Larkin R S 1994 Accelerated image reconstruction using ordered subsets of projection data IEEE Transactions on Medical Imaging 13 601-9

Ibanez J and Raurich J M 1982 Normal values of functional residual capacity in the sitting and supine positions Intensive Care Med 8 173-7

ICRP Publication 53, Radiation Dose to Patients from Radiopharmaceuticals, 1988, International Commission on Radiological Protection

ICRP Publication 80, Radiation dose to patients from radiopharmaceuticals: (Addendum 2 to ICRP Publication 53) 1998, International Commission on Radiological Protection

ICRP Publication 103, The 2007 Recommendations of the International Commission on Radiological Protection, 2007, International Commission on Radiological Protection

ICRU Report 44, Tissue Substitutes in Radiation Dosimetry and Measurement, 1989, International Commission on Radiation Units and Measurements

Jobse B N, Rhem R G, McCurry C A, Wang I Q and Labiris N R 2012 Imaging lung function in mice using SPECT/CT and per-voxel analysis PloS one 7 e42187

Jogi J, Ekberg M, Jonson B, Bozovic G and Bajc M 2011 Ventilation/perfusion SPECT in chronic obstructive pulmonary disease: an evaluation by reference to symptoms, spirometric lung function and emphysema, as assessed with HRCT Eur J Nucl Med Mol Imaging 38 1344-52

Knoll G F 1989 Radiation Detection and Measurement, 2nd Edition: John Wiley \& Sons)

Laga A C, Allen T C and Cagle P T 2008 Color Atlas and Text of Pulmonary Pathology, ed P T Cagle, et al.: Lippincott William \& Wilkins) p 628 
Larsson A, Mo S J, Ljungberg M and Riklund K 2010 Dopamine D2 receptor SPECT with (123)I-IBZM: evaluation of collimator and post-filtering when using model-based compensation-a Monte Carlo study Phys Med Biol 55 1971-88

Laurent F, Latrabe V, Raherison C, Marthan R and Tunon-de-Lara J M 2000 Functional significance of air trapping detected in moderate asthma European radiology 10 1404-10

Lee K W, Chung S Y, Yang I, Lee Y, Ko E Y and Park M J 2000 Correlation of aging and smoking with air trapping at thin-section CT of the lung in asymptomatic subjects Radiology 214 831-6

Ljungberg M and Strand S-E 1989 A Monte Carlo program for the simulation of scintillation camera characteristics. Comput Meth Prog Biomed 29 257-72

Monaghan P, Provan I, Murray C, Mackey D W, Van der Wall H, Walker B M and Jones P D 1991 An improved radionuclide technique for the detection of altered pulmonary permeability $J$ Nucl Med 32 1945-9

Laboratoire National Henri Becquerel, Tc-99m, Last Updated 17th of January 2012, Available from: www.nucleide.org

Nagao M, Murase K, Ichiki T, Sakai S, Yasuhara Y and Ikezoe J 2000 Quantitative analysis of technegas SPECT: evaluation of regional severity of emphysema $J$ Nucl Med 41 590-5

Norberg P, Persson H L, Carlsson G A, Bake B, Kentson M, Sandborg M and Gustafsson A 2013 Quantitative lung SPECT applied on simulated early COPD and humans with advanced COPD EJNMMI research 328

Olsson A, Arlig A, Carlsson G A and Gustafsson A 2007 Evaluation of reconstruction techniques in regional cerebral blood flow SPECT using trade-off plots: a Monte Carlo study Nucl Med Commun 28 719-25

Ozer C, Duce M N, Ulubas B, Bicer A, Tursen U, Apaydin F D, Yildiz A and Camdeviren H 2005 Inspiratory and expiratory HRCT findings in Behcet's disease and correlation with pulmonary function tests European journal of radiology 56 43-7

Parker J A, Coleman R E, Grady E, Royal H D, Siegel B A, Stabin M G, Sostman H D and Hilson A J W 2012 SNM Practice Guideline for Lung Scintigraphy 4.0 Journal of Nuclear Medicine Technology 40 57-65

Pellegrino R, Biggi A, Papaleo A, Camuzzini G, Rodarte J R and Brusasco V 2001 Regional expiratory flow limitation studied with Technegas in asthma J Appl Physiol 91 2190-8

Petersson J, Rohdin M, Sanchez-Crespo A, Nyren S, Jacobsson H, Larsson S A, Lindahl S G, Linnarsson D, Glenny R W and Mure M 2006 Paradoxical redistribution of pulmonary blood flow in prone and supine humans exposed to hypergravity Journal of applied physiology $100240-8$

Quanjer P H, Tammeling G J, Cotes J E, Pedersen O F, Peslin R and Yernault J C 1993 Lung volumes and forced ventilatory flows. Report Working Party Standardization of Lung Function Tests, European Community for Steel and Coal. Official Statement of the European Respiratory Society Eur Respir J Suppl 16 5-40 
RJ Ott M F, JW Babich and PK Marsden 1988 The physics of medical imaging, ed S Webb (London: IOP Publishing Ltd) p 142:318

Rosenfeld A and Kak A 1982 Digital picture processing. vol 1: Computer science and applied mathematics.)

Samee S, Altes T, Powers P, de Lange E E, Knight-Scott J, Rakes G, Mugler J P, 3rd, Ciambotti J M, Alford B A, Brookeman J R and Platts-Mills T A 2003 Imaging the lungs in asthmatic patients by using hyperpolarized helium-3 magnetic resonance: assessment of response to methacholine and exercise challenge The Journal of allergy and clinical immunology 111 1205-11

Sando Y, Inoue T, Nagai R and Endo K 1997 Ventilation/perfusion ratios and simultaneous dual-radionuclide single-photon emission tomography with krypton-81m and technetium-99m macroaggregated albumin Eur $\mathrm{J} \mathrm{Nucl}$ Med 24 1237-44

Segars W P and Tsui B M W 2001 Study of the efficacy of respiratory gating in myocardial SPECT using the new 4D NCAT phantom. In: Nuclear Science Symposium Conference Record, 2001 IEEE, pp 1536-9 vol.3

Sharma G and Goodwin J 2006 Effect of aging on respiratory system physiology and immunology Clinical interventions in aging $1253-60$

Shepp L A and Vardi Y 1982 Maximum likelihood reconstruction for emission tomography Trans Med Imag MI-1 113-22

Sovijarvi A R, Poyhonen L, Kellomaki L and Muittari A 1982 Effects of acute and long-term bronchodilator treatment on regional lung function in asthma assessed with krypton-81m and technetium-99m-labelled macroaggregates Thorax 37 516-20

Spaggiari E, Zompatori M, Verduri A, Chetta A, Bna C, Ormitti F, Sverzellati N and Rabaiotti E 2005 Early smoking-induced lung lesions in asymptomatic subjects. Correlations between high resolution dynamic CT and pulmonary function testing La Radiologia medica 109 27-39

Strålsäkerhetsmyndigheten, Vet du hur stor stråldos du får under ett år?, Last Updated April 2011, Available from: https://www.stralsakerhetsmyndigheten.se/start/

Suga K, Kawakami Y, Koike H, Iwanaga H, Tokuda O, Okada M and Matsunaga N 2010 Lung ventilation-perfusion imbalance in pulmonary emphysema: assessment with automated V/Q quotient SPECT Ann Nucl Med 24 26977

Tgavalekos N T, Musch G, Harris R S, Vidal Melo M F, Winkler T, Schroeder T, Callahan R, Lutchen K R and Venegas J G 2007 Relationship between airway narrowing, patchy ventilation and lung mechanics in asthmatics Eur Respir J 29 1174-81

Turkington T G, Wilson J M, Bowsher J E and Gilland D R 2007 Limiting iterations vs. post smoothing for noise control in PET. In: IEEE Nuclear Science Symposium Conference Record 42772 - 5,

Van Beek E J R 2004 Functional Imaging of the Chest, ed H U Kauczor and A A Bankier: Springer) 
Vander A J, Sherman J H and Luciano D S 1994 Human physiology, the mechanisms of body function, ed $\mathrm{K}$ M Prancan and J W Bradley: McGraw-Hill, Inc.)

Venegas J G, Winkler T, Musch G, Vidal Melo M F, Layfield D, Tgavalekos N, Fischman A J, Callahan R J, Bellani G and Harris R S 2005 Selforganized patchiness in asthma as a prelude to catastrophic shifts Nature $434777-82$

West J B 1991 Respiratory Physiology, the essentials: Williams \& Wilkins)

Xu J, Moonen M, Johansson A, Gustafsson A and Bake B 2001a Quantitative analysis of inhomogeneity in ventilation SPET Eur J Nucl Med 28 1795800

Xu J H, Moonen M, Johansson A and Bake B 2001b Dynamics of 'Technegas' deposited in the lung Nucl Med Commun 22 383-7

Zhang W, Wang J, Tang M, Pan J, Bai P, Lin D, Qian F, Lin F, Yang X and Zhang S 2012 Quantitative study of lung perfusion SPECT scanning and pulmonary function testing for early radiation-induced lung injury in patients with locally advanced non-small cell lung cancer Experimental and therapeutic medicine 3 631-5

Zubal I G and Harrel C R 1990 Monte Carlo determination of emerging energy spectra for diagnostically realistic radiopharmaceutical distribution. Nucl Inst Meth in Phys Res A299 544-7

Zubal I G, Harrell C R, Smith E O, Rattner Z, Gindi G and Hoffer P B 1994 Computerized three-dimensional segmented human anatomy Medical physics 21 299-302 


\section{Papers}

The articles associated with this thesis have been removed for copyright reasons. For more details about these see:

http://urn.kb.se/resolve?urn=urn:nbn:se:liu:diva-106667 